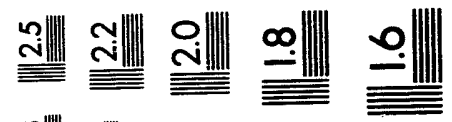

$$
\begin{aligned}
& \text { 期期 }
\end{aligned}
$$

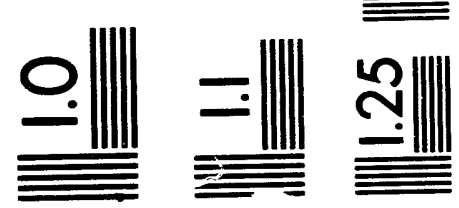



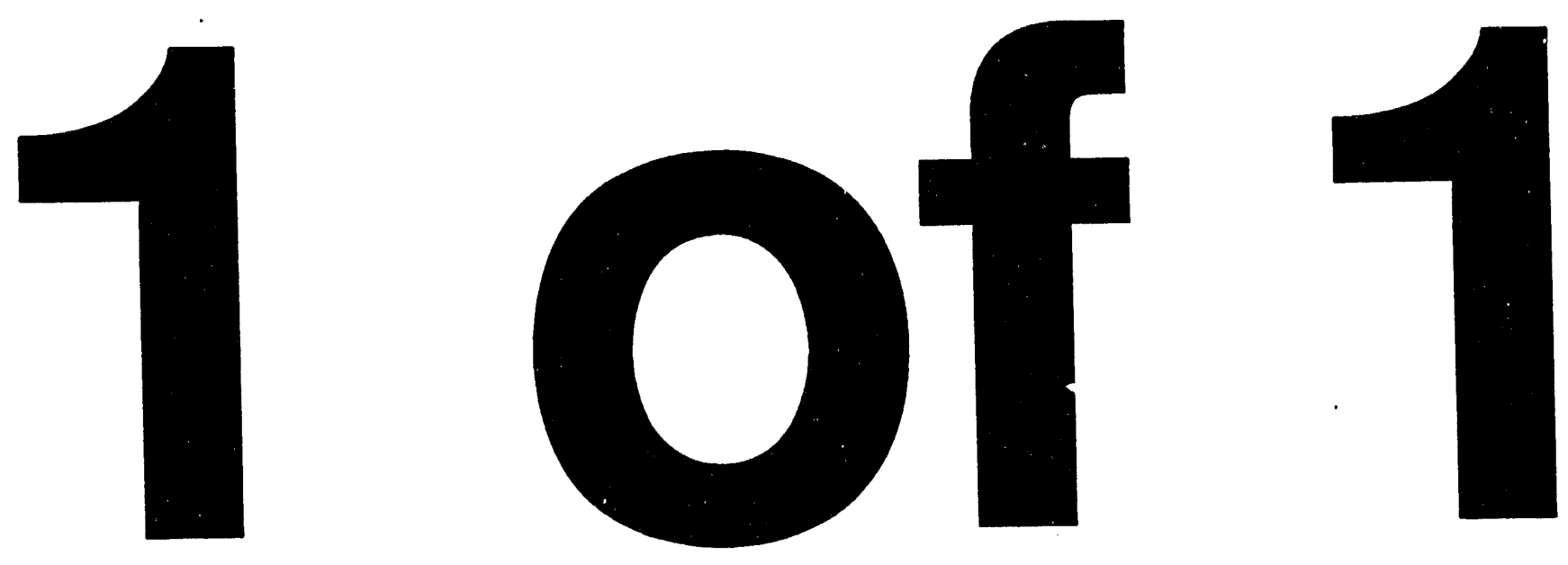


$$
\text { IS -T - } 1673
$$

\title{
Performance Analysis of Memory Hierarchies in High Performance Systems
}

\author{
by Agrawel Yogesh
}

thesis submitted to Iowa State University

Ames Laboratory, U. S. DOE

Iowa State University

Ames, Iowa 50011

Date Transmitted: July 1993

\author{
Prepared for the U. S. Department of Energy \\ Under contract no. W-7405-eng-82.
}

\section{DISCLAIMER}

\begin{abstract}
This report was prepared as an account of work sponsored by an agency of the United States Government. Neither the United States Government nor any agency thereof, nor any of their employees, makes any warranty, express or implied, or assumes any legal liability or responsibility for the accuracy, completeness, or usefulness of any information, apparatus, product, or process disclosed, or represents that its use would not infringe privately owned rights. Reference herein to any specific commercial product, process, or service by trade name, trademark, manufacturer, or otherwise does not necessarily constitute or imply its endorsement, recommendation, or favoring by the United States Government or any agency thereof. The views and opinions of authors expressed herein do not necessarily state or reflect those of the United States Government or any agency thereof.
\end{abstract}


TABLE OF CONTENTS

ACKNOWLEDGEMENTS $\ldots \ldots \ldots \ldots \ldots \ldots$ viii

CHAPTER 1. INTRODUCTION $\ldots \ldots \ldots \ldots \ldots \ldots$

CHAPTER 2. THE VIRTUAL MACHINE $\ldots \ldots \ldots \ldots \ldots \ldots$

The DLX Architecture $\ldots \ldots \ldots \ldots \ldots \ldots$

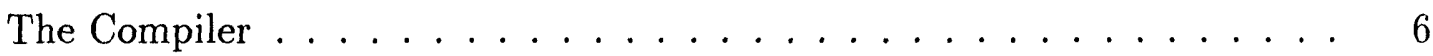

The Linker and Librarian $\ldots \ldots \ldots \ldots \ldots$

The dlxsimgc Simulator $\ldots \ldots \ldots \ldots \ldots \ldots \ldots$

CHAPTER 3. TESTING MEMORY REFERENCE COUNT AS

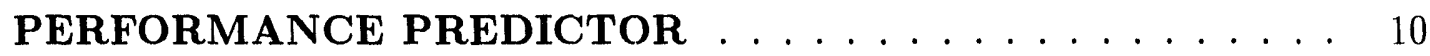

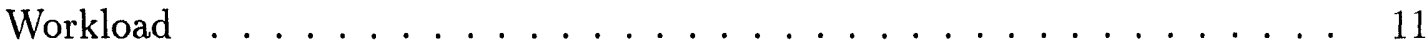

Results of Experiments . . . . . . . . . . . . . . . . . . . 12

Frequency of Accesses $\ldots \ldots \ldots \ldots \ldots \ldots \ldots$

Performance Prediction . . . . . . . . . . . . . . . . 13

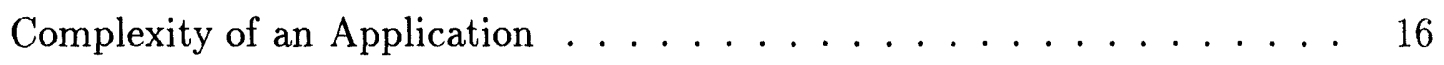

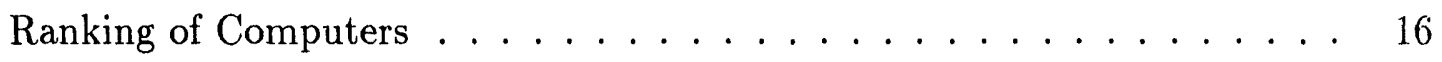

CHAPTER 4. A MODEL FOR HIGH LOCALITY PARALLEL

SYSTEMS $\ldots \ldots \ldots \ldots \ldots \ldots \ldots \ldots \ldots$ 
The Reuse Ratio . . . . . . . . . . . . . . . . . . . . . . . . 19

The Reuse Balance Point of Machines . . . . . . . . . . . . . . . . . . . . . 21

Predicting Performance. . . . . . . . . . . . . . . 21

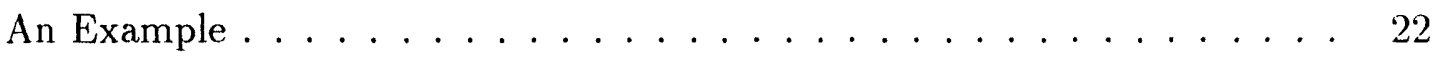

CHAPTER 5. COMPILER OPTIMIZATIONS . . . . . . . . 26

Introduction . . . . . . . . . . . . . . . . . . 26

Loop Fusion . . . . . . . . . . . . . . . . . . . 27

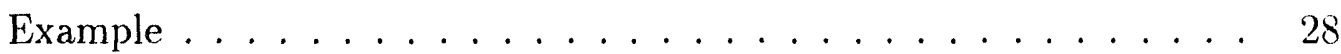

Loop Distribution . . . . . . . . . . . . . . . . . . . . . . . . 29

Example .......................... 29

Loop Interchange . . . . . . . . . . . . . . . . . . 31

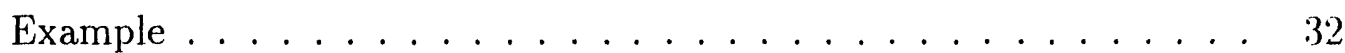

Strip Mine and Unroll . . . . . . . . . . . . . . . . . . . . . 33

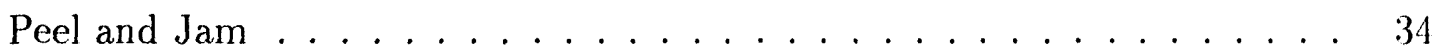

Loop Skew and Interchange . . . . . . . . . . . . . . . 35

Example ............................ 37

Blocking Transformations . . . . . . . . . . . . . 38

Strip Mine and Interchange . . . . . . . . . . . . . . . 39

Unroll and Jam . . . . . . . . . . . . . . . . . . 41

CONCLUSIONS ....................... 43

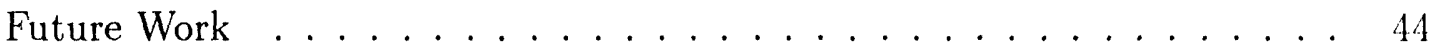

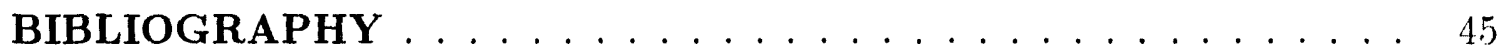

APPENDIX A. DLX STANDARD INSTRUCTION SET . . . . . . 48 


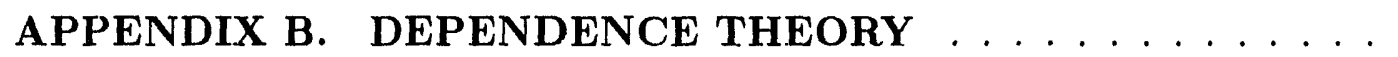

APPENDIX C. PROGRAM TO DETERMINE MEMORY BAND-

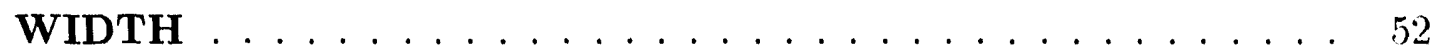




\section{LIST OF TABLES}

Table 1.1: Peak MFLOPS as performance predictor . . . . . . . . 2

Table 1.2: Effectiveness of ranking based on peak MFLOPS . . . . . . 3

Table 1.3: Peak Memory Bandwidth as performance predictor . . . . . . 3

Table 1.4: Effectiveness of ranking based on peak memory bandwidth . 4

Table 3.1: Loads and stores as a fraction of total instructions . . . . . 12

Table 3.2: Empirical memory bandwidth of the machines . . . . . 13

Table 3.3: $\quad$ Predicted and actual times on the nCUBE . . . . . . . 14

Table 3.4: Predicted and actual times on the Sun 4/370 . . . . . . 14

Table 3.5: $\quad$ Predicted and actual times on the Dec 5000/200 . . . . . 15

Table 3.6: Predicted and actual times on the HP 9000/750 . . . . . 15

Table 3.7: $\quad$ Predicted and actual times on the iPSC/860 . . . . . 15

Table 3.8: Effectiveness of ranking based on empirical memory band-

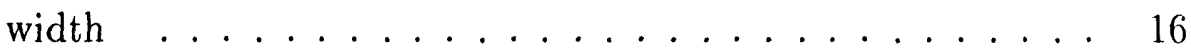

Table 4.1: The reuse ratio of some important computational kernels . . 20 


\section{LIST OF FIGURES}

Figure 4.1: Multi-level memory hierarchies . . . . . . . . . . . . . 19

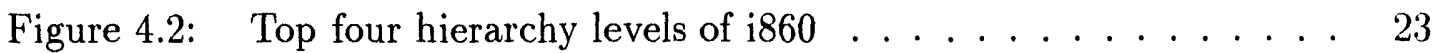

Figure 4.3: Matrix Multiplication with a constant reuse . . . . . . . . 24

Figure 4.4: Matrix Multiplication with a high reuse . . . . . . . . . . 24

Figure 5.1: Loop Fusion . . . . . . . . . . . . . . . . 27

Figure 5.2: Example of Loop Fusion . . . . . . . . . . . . . . . . . 28

Figure 5.3: Loop Distribution . . . . . . . . . . . . . . . . . . 29

Figure 5.4: Example of Loop Distribution . . . . . . . . . . . . . 30

Figure 5.5: Loop Interchange . . . . . . . . . . . . . . . . . 31

Figure 5.6: Example of Loop Interchange . . . . . . . . . . . . . . 32

Figure 5.7: Strip Mining . . . . . . . . . . . . . . . 33

Figure $5.8:$ Unrolling . . . . . . . . . . . . . . 33

Figure 5.9: Peel and Jam . . . . . . . . . . . . . . 35

Figure $5.10:$ Original Loop $\ldots \ldots \ldots$. . . . . . . . . . . 36

Figure 5.11: Skewed Loop . . . . . . . . . . . . . . . . . 36

Figure 5.12: Loop after skewing and interchanging $\ldots \ldots \ldots$. . . . . 37

Figure 5.13: Example of Loop Skew and Interchange . . . . . . . . . 37

Figure 5.14: Strip Mine and Interchange . . . . . . . . . . . . . . 39 


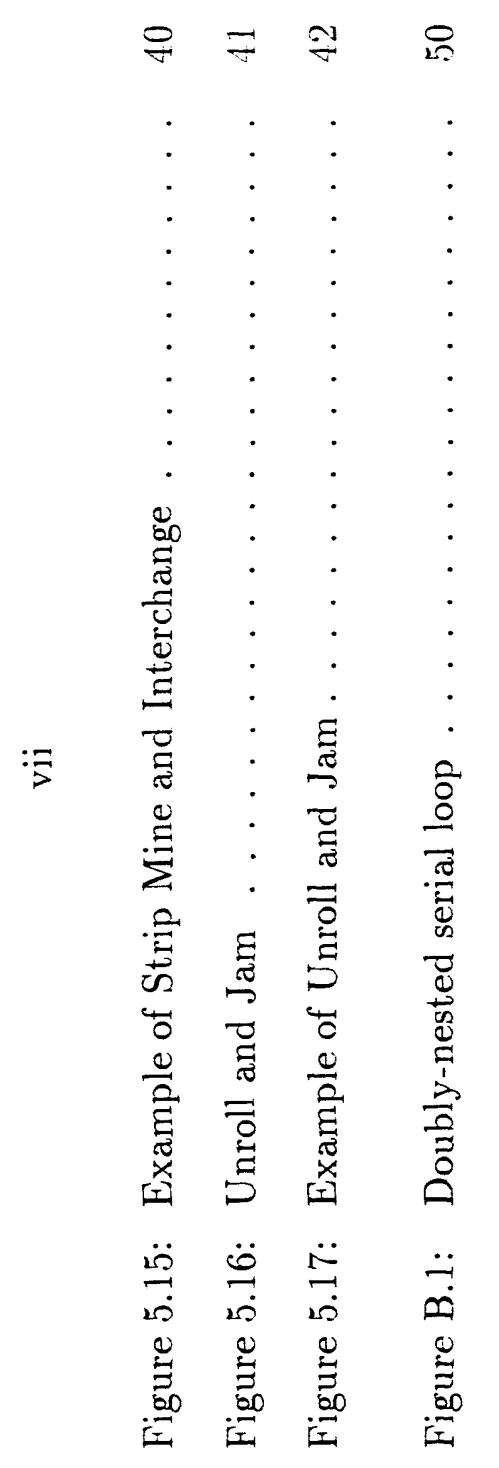




\section{ACKNOWLEDGEMENTS}

I would like to thank Dr. John Gustafson for guiding me throughout the course of my research. Without his constant help and encouragement, this work wouldn't have taken its present shape. I wish to thank Dr. Kelvin Nilsen for his on-line help and helpful discussions as my co-adviser. I would also like to thank Dr. Charles Wright for agreeing to be on my committee and for his valuable suggestions. Thanks are due to my fellow students and the staff at the Scalable Computing Laboratory, including but not limited to: Michael Carter, Joe Metzger, Srinivas Aluru and Venu Padakanti. A special note of thanks is due to Vishal Verma for helping me with the formatting of the thesis. I also wish to thank my parents for their encouragement and moral support throughout the course of my study. This research is supported in part by the Applied Mathematical Sciences Program of the Ames Laboratory which is operated for the U.S. Department of Energy under contract No. W-7405-ENG-82. The Department of Energy has assigned this document the number IS-T 1673. 


\section{CHAPTER 1. INTRODUCTION}

The potential of high-performance systems is generally limited by the bandwidth between processors and memory. Hardware has now reached the point where almost every computer from Intel and Motorola microprocessors to the CRAY 3 is limited by memory bandwidth and latency, not arithmetic. The supercomputing community has had to deal with the issue of memory-starved processors since the late 1970's. Now, the long predicted limits to conventional computer architectures have arrived at all levels, and the issue is becoming visible even in personal workstations. The consequence is a revolution in our programming models. We need new algorithms $[10,11]$, better compiler optimizations $[25,27]$ and certainly different models of "work" and "performance" [14]. Unfortunately, we still tend to operate under the assumption that "floating point operations" defines the work of an algorithm, and that "Peak MFLOPS" correlates with "Sustained MFLOPS" on most computers. As a first approximation, many people look at the peak MFLOPS rate of a computer, downgrade it to some fraction like $10 \%$, and guess that this predicts the performance they will

get on their application. This might have worked as recently as 1980 , but such an estimate will have very poor accuracy on current computers, especially supercomputers. Counterexamples that illustrate this are not hard to find. To use a recent example that compares four very different computers, consider the results from the 
EP Monte Carlo simulation (Table 1.1) taken from the NAS Parallel Benchmarks [4]:

There is no pattern in the results in the last row. All except the nCUBE use pipelined vector arithmetic. All except the CRAY are highly parallel. This benchmark does almost no communication, so interprocessor speed ratings can be neglected.

The nCUBE 2 exceeds peak speed because 64-bit floating point multiply-add operations determine the rated MFLOPS, but the benchmark involves roots, multiplies modulo $2^{46}$, and logarithms counted as 12,19 and 25 operations. The NAS weights are apparently based on CRAY Y-MP measurements. Since the nCUBE 2 does those things in less than 12,19 and 25 times the time for a floating-point multiply or add, it exceeds its "peak speed".

We also notice from Table 1.1 that the peak MFLOPS ranking of the four computers is almost the reverse of the actual MFLOPS ranking. This is clearly shown in Table 1.2. Based on peak MFLOPS, nCUBE 2 was at the bottom of the list, yet it is ranked at the top based on the actual MFLOPS.

Table 1.1: Peak MFLOPS as performance predictor

\begin{tabular}{||l|r|r|r|r||}
\hline & CRAY Y-MP/8 & CM-2 & iPSC/860 & nCUBE 2 \\
\hline EP Monte Carlo MFLOPS & 1104 & 436 & 362 & 2605 \\
\hline "Peak" rated MFLOPS & 2667 & 15000 & 7680 & 2409 \\
\hline Fraction of peak & $\mathbf{0 . 4 1 4}$ & $\mathbf{0 . 0 2 9}$ & $\mathbf{0 . 0 4 7}$ & $\mathbf{1 . 0 7}$ \\
\hline
\end{tabular}

A partial resolution for this case appears if we use "peak memory bandwidth," the rate at which all processors can use main memory (not cache) under ideal conditions (Table 1.3).

The bottom line is consistent enough to suggest that peak memory bandwidth 
Table 1.2: Effectiveness of ranking based on peak MFIOPS

\begin{tabular}{||l|c|c||}
\hline Computer & $\begin{array}{c}\text { Peak MFLOPS } \\
\text { Ranking }\end{array}$ & $\begin{array}{c}\text { Actual MFLOPS } \\
\text { Ranking }\end{array}$ \\
\hline CM-2 & 1 & 3 \\
\hline iPSC/860 & 2 & 4 \\
\hline CRAY Y-MP/8 & 3 & 2 \\
\hline nCUBE 2 & 4 & 1 \\
\hline
\end{tabular}

Table 1.3: Peak Memory Bandwidth as performance predictor

\begin{tabular}{||l|r|r|r|r||}
\hline & CRAY Y-MP/8 & CM-2 & iPSC/860 & nCUBE 2 \\
\hline EP Monte Carlo MFLOPS & 1104 & 436 & 362 & 260.5 \\
\hline Bandwidth, GB/s & 42.7 & 25 & 20 & 82 \\
\hline Ratio & $\mathbf{2 6}$ & $\mathbf{1 7}$ & $\mathbf{1 8}$ & $\mathbf{3 1}$ \\
\hline
\end{tabular}

instead of peak MFLOPS is a better simplistic predictor for this application. Also, the ranking based on the peak memory bandwidth matches perfectly with the actual MFLOPS ranking, as shown in Table 1.4. This is the main motivation behind this thesis. If peak MFLOPS correlated even weakly with observed rankings of computers, it would still be a practical tool for predicting performance. Unfortunately, in practice, it often fails to correctly rank computers. What happens is that the memory speed or processor communications (to mass storage or to other processors) can be limiting, depending on the application. Another recent example is the original Intel iPSC/860. Although rated at 60 MFLOPS in double precision, the bandwidth is only 160 MBytes per second. This limits the speed for many applications to about 6.7 MFLOPS.

This thesis studies memory bandwidth as a performance predictor of programs. 
Table 1.4: Effectiveness of ranking based on peak memory bandwidth

\begin{tabular}{||l|c|c||}
\hline Computer & $\begin{array}{c}\text { Peak MFLOPS } \\
\text { Ranking }\end{array}$ & $\begin{array}{c}\text { Actual MFLOPS } \\
\text { Ranking }\end{array}$ \\
\hline nCUBE 2 & 1 & 1 \\
\hline CRAY Y-MP $/ 8$ & 2 & 2 \\
\hline CM-2 & 3 & 3 \\
\hline iPSC $/ 860$ & 4 & 4 \\
\hline
\end{tabular}

The focus of this work is on computationally intensive programs. These programs are the most likely to access large amounts of data, stressing the memory system. Computationally intensive programs are also likely to use highly optimizing compilers to produce the fastest executables possible. Methods to reduce the amount of data traffic by increasing the average number of references to each item while it resides in the cache are explored. Increasing the average number of references to each cache item reduces the number of memory requests.

Chapter 2 describes the DLX architecture. This is the architecture on which all the experiments were performed. Chapter 3 studies memory moves as a performance predictor for a group of application programs. Chapter 4 intrcduces a model to study the performance of programs in the presence of memory hierarchies. Chapter 5 explores some compiler optimizations that can help increase the references to each iterr while it resides in the cache. 


\section{CHAPTER 2. THE VIRTUAL MACHINE}

\section{The DLX Architecture}

This project utilized the hypothetical DLX processor of Hennessy and Patterson [16], which is intended to be representative of a contemporary RISC architecture. It utilizes a small orthogonal instruction set with a single base-register-plus-offset addressing mode.

It has thirty-two 32-bit general purpose registers. Additionally, there are a set of floating point registers, which can be used as 32 single precision (32-bit) registers, or as even-odd pairs holding double-precision values. Memory is byte addressable in Big Endian mode with a 32-bit address. All memory references are through loads and stores between memory and either the general purpose registers or the floating point registers.

All instructions are 32 bits and must be aligned. Instructions pass through a five-stage pipeline, and a single branch delay slot is used. There are four classes of instructions: loads and stores, ALU operations, control operations and floating point operations. Any of the general purpose or floating point registers may be loaded or stored, except that loading $R 0$ has no effect; the value of $R 0$ is always zero. All $\mathrm{ALU}$ instructions are register-register instructions. The operations include simple

arithmetic and logical operations: add, subtract, AND, OR, XOR and shifts. There 
are also compare instructions, which compare two registers and set some condition. Control is handled through a set of jumps and branches. Floating-point instructions manipulate the floating-point registers and indicate whether the operation to be performed is single or double precision. The floating point operations are add, subtract, multiply and divide. Appendix A contains a list of all operations and their meaning.

\section{The Compiler}

It was necessary to write a $\mathrm{C}$ compiler to generate code for the DLX architecture. This compiler was based on the $l c c$ front-end developed at the Princeton University. Initial experiments on small programs were made using this compiler. However, it failed to handle very large applications like SLALOM, so we used a modified version of GNU C++ compiler, ported to DLX by Dr. William Schmidt [23].

\section{The Linker and Librarian}

The DLX assembly code files produced by the compiler must be linked together to satisfy external references, eliminate collisions between local labels, add prologue and epilogue code, and construct certain global data structures. The dlxln catenating linker, supported by the dlxlib assembly code librarian, serves this purpose. Both of these were written by Dr. Kelvin Nilsen at Iowa State University.

The primary function of the linker is to catenate all needed modules together into one file, keeping a running list of unresolved references. Since the compiler uses a uniform labeling scheme for code branch points in each assembly file it generates ( $L 1, L 2$ and so forth), the linker must also renumber all of these labels.

The dlxlib librarian allows dlxln to extract assembly code modules from li- 
braries similar to the way the UNIX linker extracts object modules from object libraries. Those library routines needed to complete the test programs were compiled for DLX and collected into common libraries. When a library is specified on the dlxln command line, the linker searches that library for modules that contain any currently unresolved references. Dependencies between modules within a library are all resolved in one pass.

\section{The dlxsimgc Simulator}

The dlxsimgc simulator written primarily by Dr. Kelvin Nilsen, emulates the overall machine architecture of DLX, including the DLX processor, instruction and data caches, memory bus and the memory module. This simulator is based on the original DLX processor simulator [17], but has been extensively rewritten in $\mathrm{C}++$ to permit simulation of the interactions between the processor and the other architectural components.

The simulator is organized in a modular fashion, with each major component in the system defined as a separate object. Objects communicate with each other by calling each other's public functions. The basic unit of computation is the processor cycle, under the assumption that the CPU is the fastest component in the system. The simulator is highly tunable; well over a hundred parameters may be individually configured in order to test different possible hardware configurations and assumptions about operation latencies. The simulator also supports a debugging configuration, permitting interactive inspection of registers and memory, breakpoints on execution addresses, and watchpoints on memory addresses to be monitored for change.

dlxsimgc gathers a number of statistics to be used in performance analysis 
studies. We enhanced the simulator to report the various operations being porformed, and the data moves between the processor and the memory. Following is a short description of the meaning of each relevant statistic:

\section{total machine instructions executed}

The number of instructions actually passing through the execute stage of the DLX pipelne.

\section{total machine cycles executed}

The latency of program execution, measured in processor cycles.

\section{total number of traps executed}

The number of system calls issued during program execution.

\section{bus utilization}

The percentage of total processor cycles during which the bus was in use with a read, write or cache invalidation request.

\section{total number of fetches}

The number of operand fetches that were made during program execution.

\section{total number of stores}

The number of writes to memory during program execution. 
icache hit ratio

The ratio of instruction fetches that were satisfied by the cache to the total number of instruction fetches.

dcache hit ratio

The ratio of operand fetches that were satisfied by the cache to the total number of operand fetches.

total number of integer operations

The number of ALU operations, not including floating point operations, made during program execution.

total number of floating-point operations

The number of floating point operations executed during program execution.

total number of move operations

The number of register-to-register moves.

total number of branch operations

The number of branches and jumps taken during program execution.

total number of nops

The number of branch delay slots that were executed during program execution. 


\section{CHAPTER 3. TESTING MEMORY REFERENCE COUNT AS PERFORMANCE PREDICTOR}

A number of popular measures have been adopted in the quest for a standard measure of computer performance; almost all of them have eventually led to misleading claims and even faulty computer designs. The fallacies in using MIPS and MFLOPS as performance predictors are well known. MFLOPS, for example, were intended to measure floating point performance and are not applicable beyond scientific computing. They fail to predict the performance of compilers, for example, because compilers rarely use any floating point arithmetic.

Our premise is that the number of memory references in any program is a better predictor of its performance. 'This holds because a large fraction of the execution time of a program is devoted to fetching from and storing to the memory. For simplicity, we purposely ignore the other factors that affect the execution time: cache, floating point operations, pipelining, etc. We conjecture that a mere count of the number of fetches in a program (ignoring the cache) predicts its performance with about $\pm 50 \%$ accuracy. 'To test our idea, we carried out experiments on a group of application programs. This chapter describes the results of those experiments. 


\section{Workload}

Four programs were ported on the DLX architecture for use in these experiments. The first program is the SLALOM benchmark, developed at Ames Laboratory [15]. It solves a radiosity problem which can be found in the pioneering paper by Greenberg, Goral, et.al. on "radiosity" [13]. The problem is to find the equilibrium radiation inside a box made of diffused colored surfaces. The faces are divided into regions called "patches", the equations that determine their color are set up, and the equations are solved for red, green and blue spectral components. This represents a real, compute-intensive application that stresses the memory system.

The sfft program [23], performs a sliding discrete Fourier transform (DFI') on a file of 8-bit audio data, computing the DFT' of the last $n$ samples as each new sample arrives. The DFT of the last $n$ samples is printed every one thousand iterations. A sample input file, containing 512 samples of raw data, was used as the input test. case.

The lisp program, written by Timothy Budd, is a $\mathrm{C}++$ implementation of the basic Lisp interpreter provided as a companion to the programming languages textbook by Kamin [18]. The test case used with lisp begins by defining the relational database functions in the textbook, and then uses these functions to create a database and make several queries to it. The lisp program is an example of a program having small code size and high instruction and data locality [23].

The matmul program multiplies two $100 \times 100$ double precision matrices. Matrix multiplication forms an important computational kernel in some scientific applications and serves as a test case that has minimal memory references in relation to the amount of arithmetic. 


\section{Results of Experiments}

This section contains an analysis of the empirical results of the study. The experiments were conducted on the test programs described in the previous section and on five different architectures: nCUBE 2, DEC 5000/200, Sun 4/370, iPSC 860 and HP $9000 / 750$. The test programs were compiled by the compiler described in Chapter 2, and the generated DLX code was executed by dlxsimgc to gather the statistics.

\section{Frequency of Accesses}

The fraction of total machine instructions that were devoted to memory accesses are shown in Table 3.1. The statistics in the table were reported by dlxsimgc.

Table 3.1: Loads and stores as a fraction of total instructions

\begin{tabular}{||l|l|r|r|r|r|r||}
\hline Program & $\begin{array}{l}\text { Input } \\
\text { Size }\end{array}$ & $\begin{array}{r}\text { Total } \\
\text { instructions }\end{array}$ & $\begin{array}{r}\text { Loads \& } \\
\text { stores }\end{array}$ & $\begin{array}{r}\% \\
\text { of total }\end{array}$ & $\begin{array}{r}\text { FP } \\
\text { Operations }\end{array}$ & $\begin{array}{r}\% \\
\text { of total }\end{array}$ \\
\hline SLALOM & 100 & 43860470 & 17176235 & $39.16 \%$ & 107224 & $0.24 \%$ \\
\hline SLALOM & 500 & 165264926 & 63166298 & $38.22 \%$ & 7080293 & $4.28 \%$ \\
\hline SLALOM & 1000 & 356413320 & 136091684 & $38.18 \%$ & 16319962 & $4.57 \%$ \\
\hline lisp & - & 216301533 & 83904672 & $38.79 \%$ & 0 & $0 \%$ \\
\hline sfft & 512 Bytes & 186981716 & 91529567 & $48.95 \%$ & 957616 & $0.51 \%$ \\
\hline matmul & $100 \times 100$ & 72707848 & 18732045 & $25.76 \%$ & 3270000 & $4.5 \%$ \\
\hline
\end{tabular}

Clearly, memory accesses comprise a high fraction of the total executed machine instructions. Floating point operations comprise a small fraction of the total instructions even for compute intensive applications like SLALOM and matmul. In fact, for the lisp program, floating point operations are zero. This again illustrates the dire consequences of using MFLOPS as a performance measure. 


\section{Performance Prediction}

We conducted experiments on five different architectures to test the effectiveness of memory accesses as a performance predictor. The program in Appendix C was run with full optimization to determine the memory bandwidth on the machines. The program attempts to defeat the cache by having a stride greater than the cache size between consecutive fetches. Table 3.2 shows the bandwidth obtained on the five machines.

Table 3.2: Empirical memory bandwidth of the machines

\begin{tabular}{||l|c||}
\hline \multicolumn{1}{|c|}{ Machine } & $\begin{array}{c}\text { Memory Bandwidth } \\
\text { (in MB/s) }\end{array}$ \\
\hline nCUBE & 12.78 \\
\hline Sun $4 / 370$ & 35.71 \\
\hline Dec $5000 / 200$ & 51.28 \\
\hline HP $9000 / 750$ & 48.19 \\
\hline iPSC 860 & 50.00 \\
\hline
\end{tabular}

For each program, execution time on a machine was predicted by multiplying the number of fetches, as reported by dlxsimgc, with the memory bandwidth on that machine. The test programs were then run with full optimization on all the machines. Tables 3.3-3.7 compare the predicted execution times with the actual execution times for the test programs on each machine. 'The predicted time for sfft deviates from the actual execution time because sfft has a very high cache hit ratio, and we ignore the cache in the model. For the other programs the deviation is high only on the DEC 5000/20 and HP 9000/750. The number of fetches for all the test programs were determined by simulations on the DLX architecture. For 
simplicity, we had assumed that this also represents the number of fetches on the other architectures, on which the experiments were performed. The actual number of fetches for the test programs on the DEC 5000 and HP 9000/750 might be different. from that suggested by dlxsimgc, leading to the deviation from the actual execution time. On the Sun 4/370, matmul deviates by an error of $66.78 \%$. matmul does a. high number of floating point operations, the time for which is not accounted for, by a mere count of fetches. This leads to an execution time that is higher than that predicted by the number of fetches. The rest of the results are within an accuracy of $\pm 50 \%$, validating our conjecture.

Table 3.3: Predicted and actual times on the nCUBE

\begin{tabular}{||l|l|r|r|r|r||}
\hline Program & $\begin{array}{l}\text { Input } \\
\text { Size }\end{array}$ & Fetches & $\begin{array}{r}\text { Predicted } \\
\text { Time }(\mathrm{sec})\end{array}$ & $\begin{array}{r}\text { Actual } \\
\text { Time }(\mathrm{sec})\end{array}$ & \% Error \\
\hline \multirow{3}{*}{ SLALOM } & 100 & 12712748 & 3.979 & 3.384 & +17.58 \\
\cline { 2 - 6 } & 500 & 49303951 & 15.432 & 11.233 & +37.38 \\
\cline { 2 - 6 } & 1000 & 107225461 & 33.561 & 23.291 & +44.09 \\
\hline lisp & - & 50056157 & 15.667 & 30.231 & -48.17 \\
\hline sfft & 512 Bytes & 16527670 & 5.17 & 2.095 & +146.77 \\
\hline matmul & 100 & 16075522 & 5.031 & 8.00 & -37.11 \\
\hline
\end{tabular}

Table 3.4: Predicted and actual times on the Sun $4 / 370$

\begin{tabular}{||l|l|r|r|r|r||}
\hline Program & $\begin{array}{l}\text { Input } \\
\text { Size }\end{array}$ & Fetches & $\begin{array}{r}\text { Predicted } \\
\text { Time (sec) }\end{array}$ & $\begin{array}{r}\text { Actual } \\
\text { Time (sec) }\end{array}$ & \% Error \\
\hline \multirow{3}{*}{ SLALOM } & 100 & 12712748 & 1.423 & 1.904 & -25.26 \\
\cline { 2 - 6 } & 500 & 49303951 & 5.522 & 6.655 & -17.02 \\
\cline { 2 - 6 } & 1000 & 107225461 & 12.00 & 13.908 & -15.90 \\
\hline lisp & - & 50056157 & 5.60 & 7.578 & -26.10 \\
\hline sfft & 512 Bytes & 16527670 & 1.85 & 1.325 & +39.62 \\
\hline matmul & 100 & 16075522 & 1.80 & 5.42 & -66.78 \\
\hline
\end{tabular}


Table 3.5: Predicted and actual times on the Dec 5000/200

\begin{tabular}{||l|l|r|r|r|r||}
\hline Program & $\begin{array}{l}\text { Input } \\
\text { Size }\end{array}$ & Fetches & $\begin{array}{r}\text { Predicted } \\
\text { Time (sec) }\end{array}$ & $\begin{array}{r}\text { Actual } \\
\text { Time (sec) }\end{array}$ & \% Error \\
\hline \multirow{3}{*}{ SLALOM } & 100 & 12712748 & 0.99 & 0.51 & +94.11 \\
\cline { 2 - 6 } & 500 & 49303951 & 3.84 & 2.02 & +90.09 \\
\cline { 2 - 6 } & 1000 & 107225461 & 8.360 & 4.916 & +70.05 \\
\hline lisp & - & 50056157 & 3.904 & 2.166 & +80.24 \\
\hline sfft & 512 Bytes & 16527670 & 1.28 & 0.46 & +178.26 \\
\hline matmul & 100 & 16075522 & 1.25 & 1.57 & -20.38 \\
\hline
\end{tabular}

Table 3.6: Predicted and actual times on the HP 9000/750

\begin{tabular}{||l|l|r|r|r|r||}
\hline Program & $\begin{array}{l}\text { Input } \\
\text { Size }\end{array}$ & Fetches & $\begin{array}{r}\text { Predicted } \\
\text { Time (sec) }\end{array}$ & $\begin{array}{r}\text { Actual } \\
\text { Time (sec) }\end{array}$ & Error \\
\hline \multirow{3}{*}{ SLALOM } & 100 & 12712748 & 1.05 & 0.59 & +77.96 \\
\cline { 2 - 6 } & 500 & 49303951 & 4.09 & 1.67 & +242.00 \\
\cline { 2 - 6 } & 1000 & 107225461 & 8.89 & 3.44 & +158.43 \\
\hline lisp & - & 50056157 & 4.15 & 2.15 & +93.02 \\
\hline sfft & 512 Bytes & 16527670 & 1.37 & 0.22 & +522.72 \\
\hline matmul & 100 & 16075522 & 1.33 & 1.15 & +15.65 \\
\hline
\end{tabular}

Table 3.7: Predicted and actual times on the iPSC/860

\begin{tabular}{||l|l|r|r|r|r||}
\hline Program & $\begin{array}{l}\text { Input } \\
\text { Size }\end{array}$ & Fetches & $\begin{array}{r}\text { Predicted } \\
\text { Time (sec) }\end{array}$ & $\begin{array}{r}\text { Actual } \\
\text { Time (sec) }\end{array}$ & \% Error \\
\hline \multirow{3}{*}{ SLALOM } & 100 & 12712748 & 1.02 & 1.32 & -22.72 \\
\cline { 2 - 6 } & 500 & 49303951 & 3.94 & 3.49 & +12.89 \\
\cline { 2 - 6 } & 1000 & 107225461 & 8.58 & 7.07 & +21.35 \\
\hline lisp & - & 50056157 & 4.00 & - & - \\
\hline sfft & 512 Bytes & 16527670 & 1.32 & 0.63 & +109.52 \\
\hline matmul & 100 & 16075522 & 1.28 & 1.44 & -11.11 \\
\hline
\end{tabular}




\section{Complexity of an Application}

Curve fitting can be done on the number of fetches for various input sizes to determine the polynomial that best approximates the complexity of the application in terms of fetches. A fit on the number of fetches for SLALOM for various input sizes expresses the complexity of SLALOM in the following polynomial.

$$
169823+105458 N
$$

Similarly, when matmul was run for various input sizes we found its complexity to be:

$$
-11.64-88.90 N+20.40 N^{2}+15.80 N^{3}
$$

\section{Ranking of Computers}

Table 3.8 shows the effectiveness of memory bandwidth in ranking computers. The computers are first ranked based on their empirical memory bandwidth. This ranking is then compared against the actual observed rankings on the applications.

Table 3.8: Effectiveness of ranking based on empirical memory bandwidth

\begin{tabular}{||l|r|c|c|c|c|c|c|c||}
\hline \multirow{2}{*}{ Machine } & $\begin{array}{c}\text { Empirical } \\
\text { Bandwidth } \\
\text { (in MB/s) }\end{array}$ & \multirow{2}{*}{ Ranking } & \multicolumn{5}{|c|}{ Actual Ranking on Applications } \\
\cline { 4 - 10 } & & $\begin{array}{c}\text { slalom } \\
100\end{array}$ & $\begin{array}{c}\text { slalom } \\
500\end{array}$ & $\begin{array}{c}\text { slalom } \\
1000\end{array}$ & lisp & sfft & matmul \\
\hline DEC 5000/200 & 51.28 & 1 & 1 & 2 & 2 & 1 & 2 & 3 \\
\hline iPSC $/ 860$ & 50.00 & 2 & 3 & 3 & 3 & - & 3 & 2 \\
\hline HP 9000/750 & 48.19 & 3 & 2 & 1 & 1 & 2 & 1 & 1 \\
\hline Sun 4/370 & 35.71 & 4 & 4 & 4 & 4 & 3 & 4 & 4 \\
\hline nC,UBE & 12.78 & 5 & 5 & 5 & 5 & 4 & 5 & 5 \\
\hline
\end{tabular}


The peak bandwidth of DEC $5000 / 750$ and HP $9000 / 750$ are very close and they are consistently at the top two positions on most of the applications. iPSC/860 is at the third position on all applications except matmul. Sun 4/370 and nCUBE whose bandwidth are much less as compared to the other machines, occupy the two lowest positions on all the applications. 


\section{CHAPTER 4. A MODEL FOR HIGH LOCALITY PARALLEL SYSTEMS}

In this chapter, we present a simple quantitative model for high-locality parallel systems [7]. High-locality parallelism is composed of three components:

1. algorithms that block well into caches.

2. efficient parallel coordination to extract a large fraction of a system's peak memory bandwidth.

3. enough parallel processors to saturate memory.

The goal is to convert memory bandwidth into delivered performance. As we have seen earlier, memory bandwidth, not processing power, is the limiting factor in modern systems. We assurne computations that are huge and embarassingly parallel.

We model a system as a multi-level memory hierarchy (Figure 4.1). The top levels are fast but small in capacity. The bottom levels are slow but capacious. Only levels 1-3, comprising of the arithmetic units, registers and cache, can fit inside a chip using current technology. Levels 4 and 5 are the two types of solid state memory. Levels 6 and 7 are the two dominant types of mechanical storage.

Level $m$ of this hierarchy is characterized by two parameters: its size $S_{m}$, and

the bandwidth $B_{m+1}$ available from the next slower level $m+1$. If a layer in the 


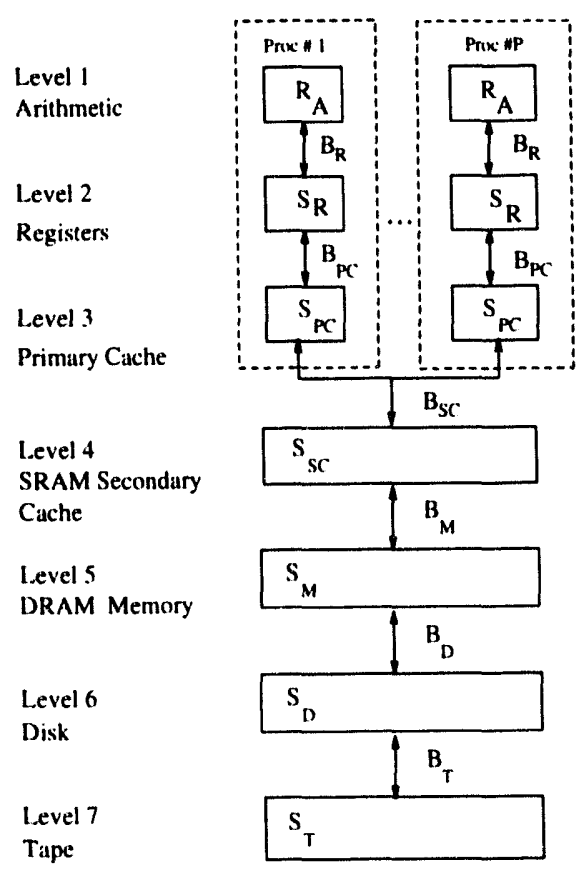

Figure 4.1: Multi-level memory hierarchies

hierarchy is implemented as multiple private caches, then $P+1$ is the aggregate bandwidth from the next slower layer, but $S_{m}$ is the size of each individual cache.

\section{The Reuse Ratio}

We define reuse for a level as the number of floating point operations per nonlocal access. Let $n_{f}$ be the number of floating point operations and let $d_{m}$ be the number of data transfers from level $m+1$ to level $m$. Then reuse for level $m$ is $n_{f} / d_{m}$. The LAPACK literature uses this ratio of work divided by data traffic without explicitly naming it. 
The first application of the reuse ratio is to quantify the compute intensity of algorithms. A reuse of 1 means that for every floating point operation, an algorithm requires one data point. Low reuse indicates that the algorithm is memory bound whereas a high reuse indicates that the algorithm is compute bound. To make things clearer we consider some examples.

A scalar floating-point multiply does one operation, and references three data points, for a reuse of $1 / 3$. The SAXPY vector operation does $Y=Y+a * X$, where $X$ and $Y$ are $N$ element vectors, and $a$ is a scalar. SAXPY does $2 N$ operations and consumes, $3 N$ data points, for a constant reuse of $2 / 3$, regardless of the vector length. Finally, consider a matrix multiply-add: $Y=Y+A * Y$, where $A, X$ and $Y$ are $N \times N$ matrices. A matrix multiply-add does $2 N^{3}$ operations, and requires $4 N^{2}$ operand references, for a reuse of $0.5 \mathrm{~N}$. Table 4.1 lists the reuse of some important computational kernels.

Table 4.1: The reuse ratio of some important computational kernels

\begin{tabular}{||l|c|c|c||}
\hline Kernel & FP Operations & Data Words & $\begin{array}{c}\text { Reuse } \\
\text { (level 1) }\end{array}$ \\
\hline Vector Add & $1 \mathrm{~N}$ & $3 \mathrm{~N}$ & 0.33 \\
\hline Vector Multiply & $1 \mathrm{~N}$ & $3 \mathrm{~N}$ & 0.33 \\
\hline SAXPY & $2 \mathrm{~N}$ & $3 \mathrm{~N}$ & 0.67 \\
\hline Dot Product & $2 \mathrm{~N}$ & $2 \mathrm{~N}$ & 1 \\
\hline GAXPY & $2 \mathrm{NM}$ & $1 \mathrm{NM}$ & 2 \\
\hline Matrix Multiply & $2 N^{3}$ & $4 N^{2}$ & $0.5 \mathrm{~N}$ \\
\hline
\end{tabular}

For a memory hierarchy to be successful, the algorithm in question should have a high reuse; ideally reuse increases with the caching size. The reuse of matrix multiply. grows as the square root of the memory hierarchy level size [10]. Moving up from an $8 \mathrm{~KB}$ primary cache to a 2 (iB memory, increases reuse by a factor of 512. The 
reuse of FFTs, on the other hand, increases only as the logarithm of the level size. Increasing the size by a factor of $256 \mathrm{~K}$ improves locality by only a factor of three [7].

\section{The Reuse Balance Point of Machines}

The second application of the reuse ratio is to quantify the balance between the compute and data movement capahilities of a computer system. We can compare a machine's reuse balance point with the reuse of a proposed application. Algorithms with a higher reuse than the machine's balance point, will be compute limited, and algorithms with a lower reuse than the machine's balance point will be memorylimited.

For example, on a vector supercomputer, like the CRAY-X/MP and Y/MP, each processor can perform two floating-point operations and can access three data points from memory every clock. The reuse balance point is $2 / 3$ operations per point. This matches well with the reuse of simple vector operations, but is compute-limited for matrix operations. A second example is the Silicon Graphics Power Series. Up to eight 13 MFLOPS processors share a $64 \mathrm{Mbytes} / \mathrm{s}$ memory bus. In 64-bit precision, this is a reuse balance point of $104 / 8=13$ operations per point. This computer needs the higher reuse of matrix operations to deliver its best performance.

\section{Predicting Performance}

The third application of the reuse ratio is to estimate the performance of a fully saturated memory hierarchy. Level $m$ of a memory hierarchy limits the maximum possible performance to:

$$
\text { Performance }_{m}=\text { Reuse }_{m} * \text { Bandwidt }_{m+1}
$$


As an example, consider an algorithm that exhibits reuse of 20 operations per point, and an available memory bandwidth of 140 million points per second. For such a system, the memory-limited performance is 2,800 MrLOPS.

For a multi-level hierarchy, the performance is the minimum of that imposed by all of the levels. The lower levels may be ignored when all the relevant data fits in one of the higher levels. For example, if all the data fits in memory, the effects of disk and tape may be ignored.

Given the reuse-based performance estimate, we can estimate how many processors are needed to saturate memory. An individual processor's internal performance estimate, Perf cpu, can be obtained from hand coding, compiling, or by examining the processor's asymptotic functional-unit capabilities.

The number of processors $P_{m s a t}$ needed to saturate the memory is,

$$
P_{m s a t}=\left\lceil\operatorname{Per} \int_{m r m} / \operatorname{Per} \int_{c p u}\right\rceil
$$

where Per $\int_{m e m}$ is the memory-limited performance. If the system cannot physically hold $P_{m s a t}$ processors, then the performance is compute-limited instcad of being memory-limited.

\section{An Example}

The choice of an algorithm can have a significant effect on the reuse ratio. Wo illustrate this with two matrix multiplication algorithms. We also illustrate the performance of one of the algorithms on the i\$60 microprocessor.

The i860 microprocessor can complete one multiply-ard in two clock cycles. The clock rate is $40 \mathrm{MHz}$. Therefore, 


$$
\operatorname{Per} f_{c p u}=\frac{1 m u l+1 a d d}{2 \text { clocks }} * 40 M h z=40 \text { MFLOPS }
$$

The primary cache holds 1,024 64-bit words. The off-chip bandwidth to level four of the memory hierarchy (Figure 4.2) is a 32-bit address/64-bit data bus that cycles at $20 \mathrm{Mhz}$, yielding $160 \mathrm{MB} / \mathrm{s}$.

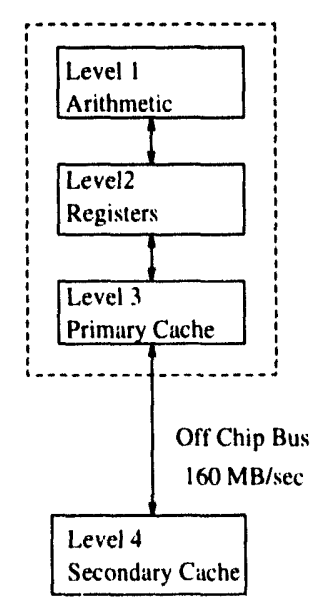

Figure 4.2: Top four hierarchy levels of i860

Now, consider the two matrix multiplication algorithms shown in Figures 4.3 and 4.4. The GAXPY formulation, popular on vector machines, is shown in Figure 1.3. An $R$-sized strip of the result is accumulated in the vector-registers, where $R$ is the vector register length. The II and $\mathrm{J}$ loops can run in parallel and the I loop vectorizes. Therefore the reuse is:

$$
\begin{aligned}
\frac{2 N R}{2 R+N R+N} & \frac{2 R}{2 R / N+R+1}
\end{aligned}
$$




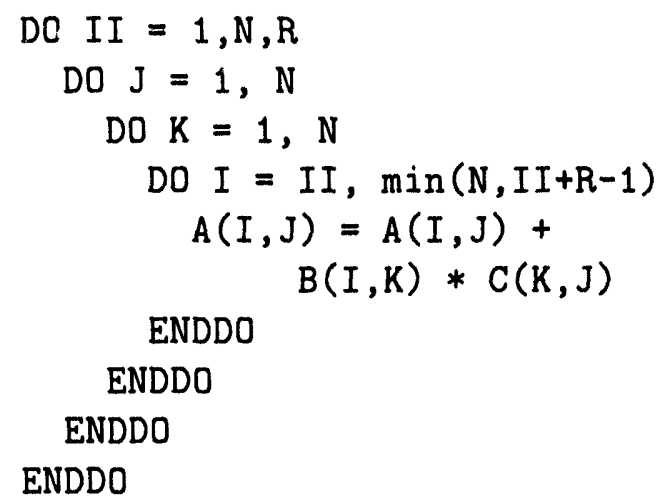

Figure 4.3: Matrix Multiplication with a constant reuse

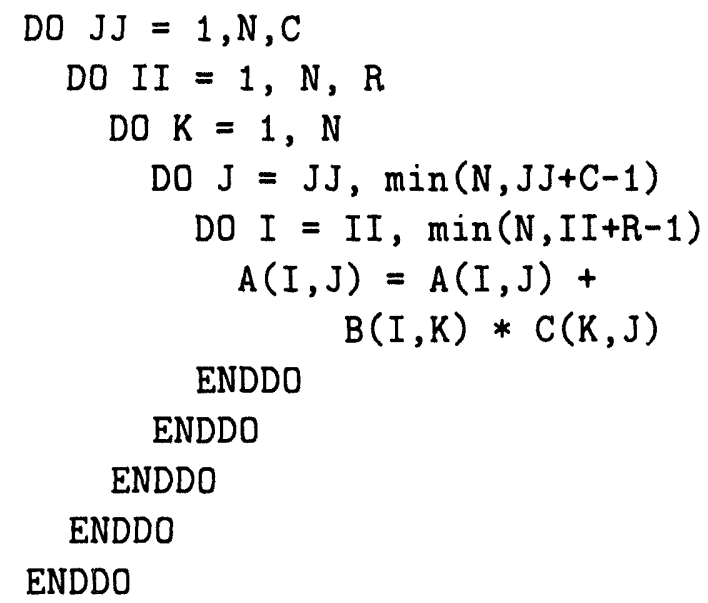

Figure 4.4: Matrix Multiplication with a high reuse 
For $N>R, R / N \approx 0$

Therefore,

$$
\text { reuse } \quad=\frac{2 R}{R+1} \approx 2 \text { for } R>>1
$$

Thus, the reuse is independent of the size of the matrix.

The block matrix formulation, shown in Figure 4.4, generalizes the strip mining to two dimensions by adding a $\mathrm{JJ}$ loop. The reuse in this case is:

$$
\begin{aligned}
& =\frac{2 N R C}{N R+N C+2 R C} \\
& \approx \frac{2 R C}{R+C}, \text { when } N>>R \text { or } C
\end{aligned}
$$

The reuse is largest when $R=C . R=C=30$ is the largest submatrix size that can fit in the i860's cache, and we look at some performance figures for these values of $R$ and $C$.

$$
\begin{aligned}
\text { Reuse }_{\text {chip }} & =\frac{2 * 30^{2}}{30+30}=30 \\
{\text { Per } f_{\text {chip }}} & =\text { Reuse }_{\text {chip }} * \text { Bandwidth }_{\text {bus }} \\
& =30 * 20 \\
& =600 \mathrm{MFLOPS} \\
& =\left\lceil\text { Per } f_{\text {chip }} / \text { Per } f_{\text {cpu }}\right\rceil \\
P_{\text {sat }} & =600 / 40 \\
& =15
\end{aligned}
$$




\section{CHAPTER 5. COMPILER OPTIMIZATIONS}

\section{Introduction}

In this chapter we provide a survey of some compiler optimizations that can improve the performance of caches. If the program can be transformed into a version that makes better use of the cache, then the number of requests to memory can be reduced, improving the execution time. Over the years, researchers have developed optimizing compilers that apply a number of transformations reordering computation without changing the results. A transformation that reduces the amount of memory used between the endpoints of a dependence edge may change a reference from a miss to a hit. A brief introduction to dependence theory is given in Appendix B.

The transformations examined here are loop fusion, loop distribution, loop inlerchange, strip mine, unroll, two combinations of transformations peel and jam, loop skew and interchange and two blocking transformations (strip mine and interchange and unroll and jam). All of these transformations are loop based transformations. and can potentially change the order of execution of many statements. Transformations are safe when no dependence is reversed by the transformation $[1,24] . \Lambda$ dependence is said to be reversed by a transformation when the source and sink of the dependence edge are interchanged. 
during execution.

Eventually, information about memory requirements of the various regions could be used in deciding which regions are best to merge. When several regions could be merged, a good heuristic is to merge the two regions that require the least amount of memory after the merge. Memory performance information can also be used to determine the maximum parallel width during execution by estimating the amount of memory traffic produced by each processor. Any processor added after the memory is saturated will not decrease execution time.

\section{Example}

The example below (Figure 5.2) demonstrates how fusion can improve memory performance when the two loops being fused use the same data, but one execution of either loop flushes the memory.

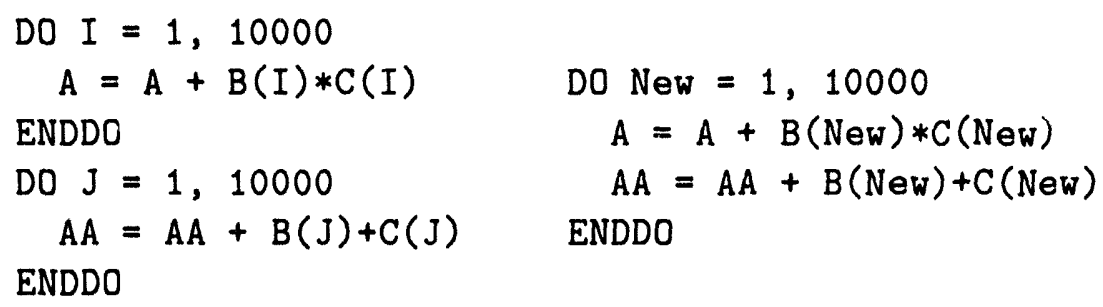

Figure 5.2: Example of Loop Fusion

Both loops in the original program use a total of 20,001 words or 80,004 bytes (single precision) of cache. A cache of less than $80 \mathrm{~K}$, such as the Alliant FX/8 and the IBM 3090 would not have either $\mathrm{B}$ or $\mathrm{C}$ present when accessed during execution of the second loop. After the loops are fused, the values of $\mathrm{B}$ and $\mathrm{C}$ are used in both 
loop bodies before being bumped from cache. The original program produced 40,000 misses and no hits. After fusion, there were 20,000 hits and only 20,000 misses. Thus, loop fusion reduced the number of misses in this example by $50 \%$.

\section{Loop Distribution}

Loop Distribution (Figure 5.3) is the inverse of loop fusion [22]. Any pairs of loops fused together can be split into the original pair of loops by distribution. After distribution, the dependence edges between iterations of a single loop body span less code than before distribution, so they may become hits. Loop distribution can also be used in a compiler to break a loop into smaller sections that are hopefully easier to vectorize and parallelize.

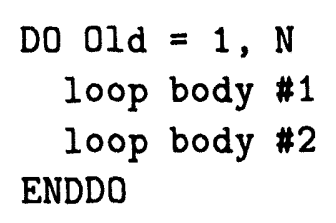

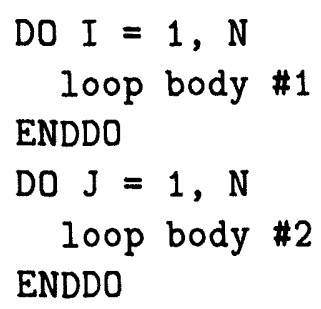

Figure 5.3: Loop Distribution

\section{Example}

The loop in Figure 5.4 demonstrates how distribution can improve memory performance when the loop being split uses more data than the size of the cache and at least one of the two new loops fits into the cache. 
Before loop distribution, each K loop uses 10,000 elements (or 40,000 bytes single precision). When both new loops are executed, a total of 80,000 bytes are touched. Since this is greater than a $64 \mathrm{~K}$ cache, the elements of $\mathrm{B}$ and $\mathrm{C}$ are pushed out before they can be used a second time. After distribution, only 40,000 bytes are used in exch loop, allowing $\mathrm{B}$ and $\mathrm{C}$ to be retrieved from cache after the first execution. Before distribution, the loops executed 2,000,000 loads (all misses) and 1,000,000 stores (all hits). After distribution, the 1,000,000 stores and 990,000 of the loads are still hits. Thus, loop distribution raised the hit ratio from $33 \%$ to $66 \%$ for this code segment.

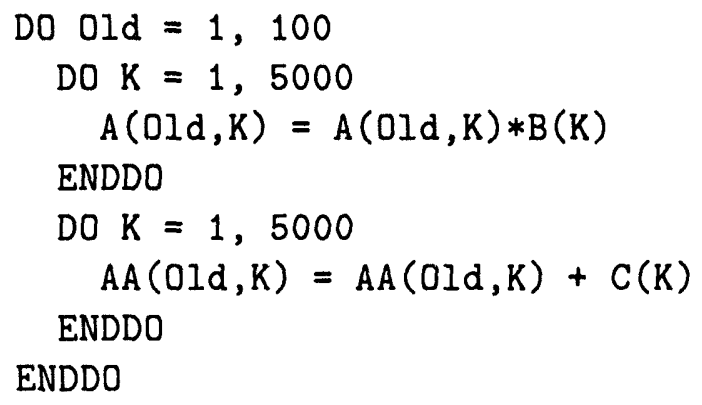

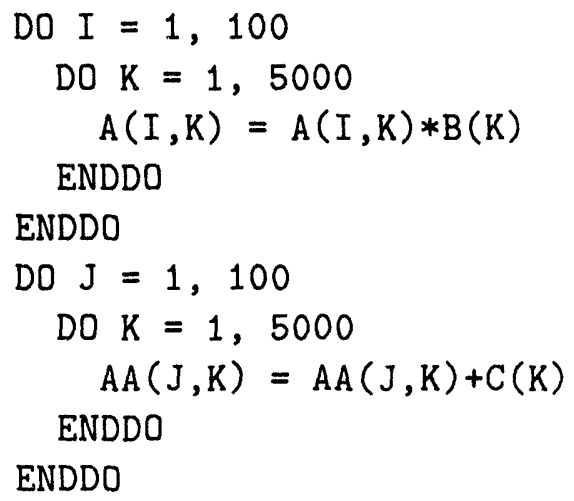

Figure 5.4: Example of Loop Distribution

The profitability of fusion on the distributed loops in this example is very negative (i.e., fusion increases the misses by 990,000). Thus, recombining parallel sections should not be done when the two original loops reuse array values in the cache and the combined loop causes the values to be replaced in the cache before being reused. 


\section{Loop Interchange}

As shown in Figure 5.5, loop interchange simply reverses the order of execution of a pair of nested loops $[2,3,22,27]$. It is used to move parallel loops out to lower levels of nesting (increasing the parallel region size) or move vectorizable loops toward higher levels of nesting based on the target hardware.

Before interchange, one iteration of the $J$ loop causes its loop body to be executed only one time, while one iteration of the $l$ loop results in $M$ executions of its loop body. After interchange, the $I$ loop requires only one iteration of its loop body, while the $J$ loop now requires $N$ iterations of its loop body. This causes the amount of memory used by an iteration of each loop to change.

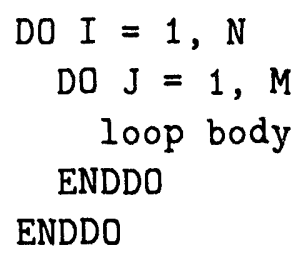

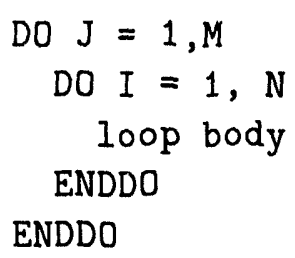

Figure 5.5: loop Interchange

Loop interchange is safe when no dependence edges are reversed by the transformation. Edges can be reversed when the direction vector associated with a reforence pair has different directions for the $\mathrm{I}$ and $\mathrm{J}$ loops (for instance $(<,>)$ ) [1] in a nest of loops indexed by I and J. Loop interchange does not change the statements exccuted during either the outer or inner loop. 


\section{Strip Mine and Unroll}

Strip mining (illustrated in Figure 5.7), groups the iterations of a loop, typically the inner loop, into larger segments $[21,22,27,29]$. 'The original loop's step size increases to accommodate the increased work being done within each of its iterations. Unrolling illustrated in Figure 5.8, also groups the iterations of a loop, but rather than forming a new inner loop, the loop body is replicated some number of times. Again, the loop step of the original loop is increased.

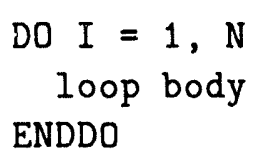

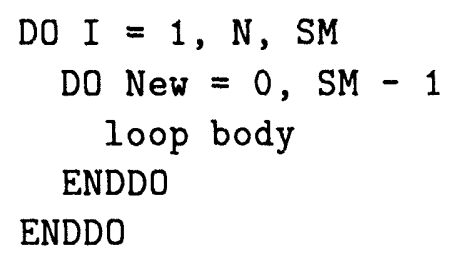

Figure 5.7: Strip Mining

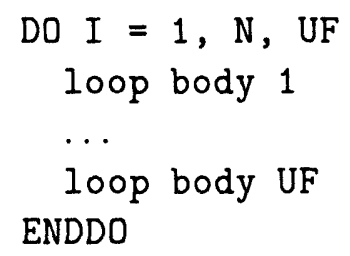

Figure 5.8: Unrolling

Thus, strip mining and unrolling are two forms of of the same transformation. Both transformations take a number of iterations and package them as a single mit that can be manipulated by other transformations. If the new loop produced by strip mining were completely unrolled, the resulting code would be identical to the 
code after unrolling the original code. Strip mining builds a new loop that contains some number of iterations; unrolling just increases the size of the already existing inner loop. The loop structure that strip mining introduces slows the resulting code slightly. Unrolling reduces the number of executed loop headers but increases the number of generated instructions.

Strip mine and unroll does not change the order of execution of the modified loop, but it can be used to increase the effectiveness of other transformations. This combination is useful in building chunks of computation that use a specific amount. of memory. By varying the size of the chunks, these transformations can be used to tune the results of other transformations. In the next sections, we show that by choosing the correct chunk size, interchange or fusion can be reliably used to improve memory performance of nested loops.

\section{Peel and Jam}

Peel and jam $[5,22]$ is a transformation that fuses loops with certain kinds of fusion preventing dependence edges. Fusion preventing edges are dependences that would be reversed by fusion. An example is when the first loop defines a value on the second iteration and the second loop uses it in its first iteration. The two loops could not be fused because the value would be used before it was defined. By poeling a number of iterations of the first loop body equal to the distance of the fusion preventing dependence, the correct relationship between the references in the two loop bodies is maintained after fusion (Figure 5.9).

In peel and jam, the first loop of a pair of loops has a small number of iterations unrolled into a prologue. The majority of the iterations are left in a loop that can be 


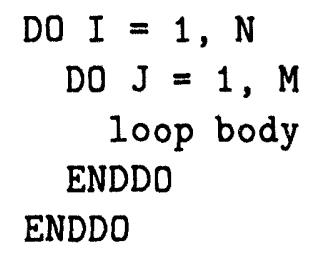

Figure 5.10: Original Loop

- replacing the lower bound of the $J$ loop, LBJ, with the expression $\left(\mathrm{LB} J \mathrm{~J}+\mathrm{I}^{*} \mathrm{f}\right)$

- replacing the upper bound of the $J$ loop, UBJ, with the expression (UBJ J I*f)

- replacing all occurrences of $\mathrm{J}$ in the loop body with the expression $\left(\mathrm{J}-\mathrm{I}^{*} \mathrm{I}\right)$

The skewed loop with a scale factor of 1 is shown in Figure 5.11. Interchanging the skewed loops requires some clever modifications to the loop limits (Figure 5.12).

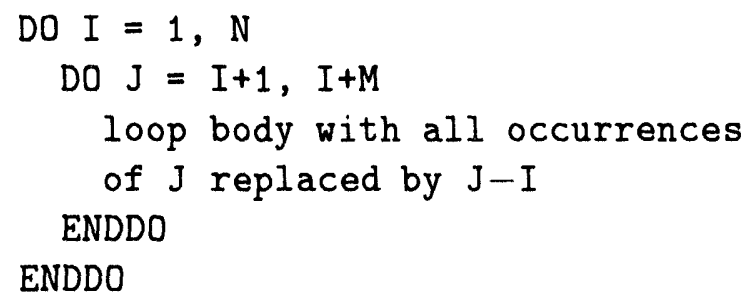

Figure 5.11: Skewed Loop

Loop skewing is always safe. 'Thus, the safety of the combination focuses on the safety of loop interchange after skewing. In reference [28], Wolfe shows that if the skew factor, $f$, is greater than or equal to the maximum distance, $d$, of any fusion preventing dependence, then loop skew and interchange is a safe reordoring transformation. 


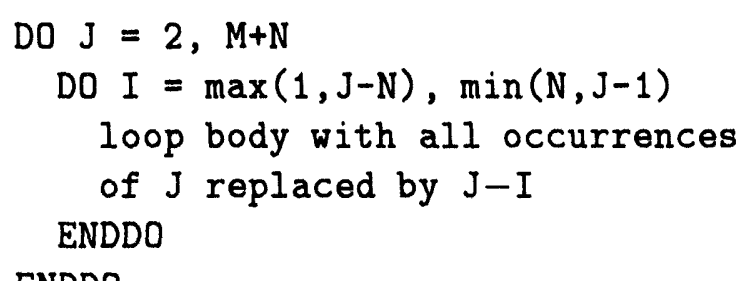

ENDDO

Figure 5.12: Loop after skewing and interchanging

\section{Example}

Data cache management can benefit from loop skewing, similar to the benefits of peel and jam. A small change to the example used in loop interchange produces the following example (Figure 5.13), which cannot be interchanged without skewing the loops.

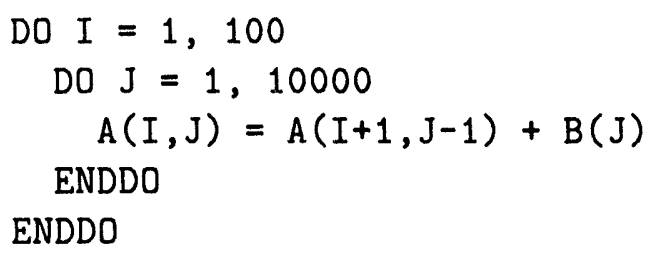

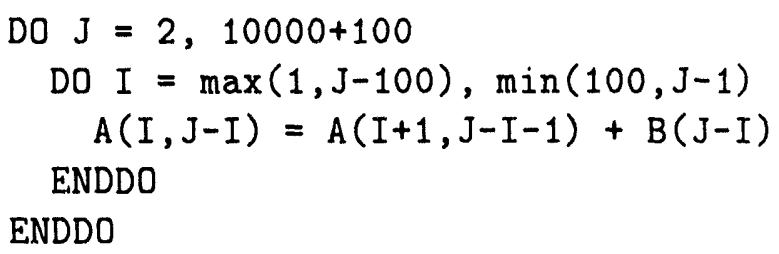

Figure 5.13: Example of Loop Skew and Interchange

Before the loop skew and interchange, every access in the loop is a miss (for a $32 \mathrm{~K}$ cache). Between iterations of the I loop, 10,000 different A's and B's are loaded, easily flushing the cache. Overall, the loop would cause 3 million misses at execution time. After loop interchange, the loop performs much better. During each iteration 
of the I loop, at most 100 values of $\mathrm{B}$ are loaded, (normally in the range J-I to $\mathrm{J}-100)$. During the next iteration of the J loop, 99 of the B's are reused and only. one is loaded. The total number of references to $B$ that are misses is 10,000 when boundary conditions are taken into account. During each iteration of the loop, the store to A uses a location that was loaded in the previous iteration. This is casy to verify by adding one to $I$ in both subscript positions $(I+I$ and $J-(I+1))$ resulting in the subscripts of the right side. Every store to $\Lambda$ will be a hit, so the only misses occur when it is loaded. Since there are 1 million elements of $A$, every iteration must bring in a new value. The total number of misses for this loop nest after loop skew and interchange is $1,010,000$, about one-third of the number of misses before transformation.

\section{Blocking Transformations}

Blocking is a well-known optimization technique for improving the effectiveness of memory hierarchies. Instead of operating on entire rows or columns of an array, blocked algorithms operate on submatrices or blocks, so that data loaded into the faster levels of the memory hierarchy are reused. Blocking has been shown to be usceful for many algorithms in linear algebra. For example, the latest version of the basic linear algebra library (BLAS 3) [9] provides high-level matrix operations to support blocked algorithms. Finding a blocking size that allows the inner loops to fit into the memory is crucial to effective blocking. 'Tuning the block size to fit into memory will allow the minimum number of misses to occur and generate the least traffic between the memory levels. 'This section describes two blocking transformations, strip mine and interchange and unroll and jam. 


\section{Strip Mine and Interchange}

Strip mine and interchange $[2,3,19,21,22,25,26,27,29]$ can improve corles such as matrix multiplication where there are references with hit ratios determined by dependences carried by the outer loop, and which are misses in spite of small distances. The goal is to strip the inner loop into pieces such that the new inner loop) fits entirely into memory. The newly created middle loop is then moved to the outer loop (Figure 5.14). The original outer loop will now allow references that have have dependence edges with small distances to be hits.

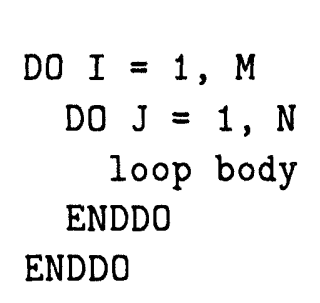

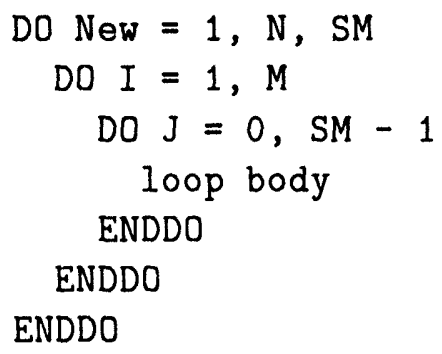

Figure 5.14: Strip Mine and Interchange

To block most effectively with strip mine and interchange, the compiler needs to calculate the optimal strip size. When the distances of dependences are relatively small, the best strip size will be the largest strip that still allows the outer loop's dependences to become hits. This moves the fewest inner loop references to the new strip mined loop. When the outer loop dependence distances are not computable, the optimal value may leave some outer loop dependences as misses. The common assumption is that dependences almost all have very short distances (less than 4 or 5). Under this assumption, the optimal strip mine width will be the width that callses 
the fewest iterations to be moved out and allows all of the outer loop dependences to become hits.

Example Strip mine and interchange is a profitable transformation when there exists a loop nest where the inner loop requires slightly more memory than is available and references are misses due to outer loop carried dependences. The number of references carried by the inner loop must be less than SM times the number of references carried by the outer loop. Strip mine and interchange substantially reduces the misses in the classic numerical problem, matrix multiplication illustrated in Figure 5.15.
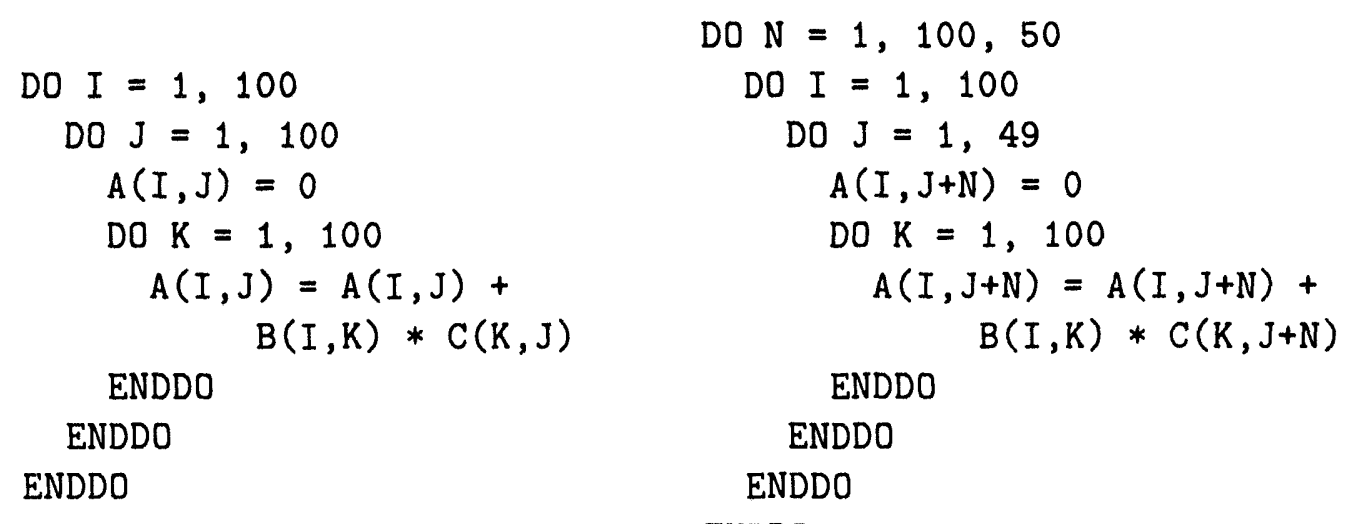

ENDDO

Figure 5.15: Example of Strip Mine and Interchange

When a $32 \mathrm{~K}$ cache is used, the references to the $\mathrm{C}$ array are misses. Combincd with the initial loads of the A and B arrays, the original program will miss an 8-way set associative cache 1,020,000 times. In the strip mine and interchange version, the $\mathrm{J}$ loop uses 5,000 elements of the C array (instead of 10,000). If the array is single 


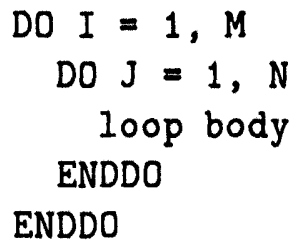

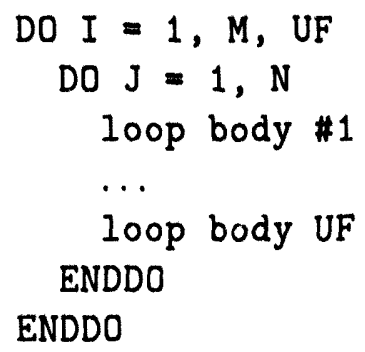

Figure 5.16: Unroll and Jam

precision, a $32 \mathrm{~K}$ cache holds the $\mathrm{C}$ array between iterations of the I loop. Each iteration of the new $\mathrm{N}$ loop requires the values to be brought back into the cache. Overall, the new program produces only 40,000 misses. For matrix multiplication, strip mine and interchange can remove up to $96 \%$ of the generated misses.

\section{Unroll and Jam}

Strip mine and interchange takes an inner loop, blocks it and moves some of the iterations to outer loops, in an effort to allow dependences carried at the outer level to become hits. Unroll and jam [6, 22] moves blocks inward (Figure 5.16), blocking the outer loop and pushing computation into the inner loop. The goal is to have dependences carried by the outer loop move to the inner loop so they can become hits. The transformation is actually unrolling followed by fusion, but the authors of reference [6] created the term "unroll and jam" to better describe the global transformation.

Both "strip mine and interchange" and "unroll and jam" block the computation into bounded regions that are sized to allow near optimal use of limited sized memory structures. The difference between the two transformations is the loop that gots 
blocked. In strip mine and interchange, the inner loop is blocked and moved outward. Unroll and jam blocks the outer loop and moves it inward.

Example Unroll and jam is profitable when there are references depending on outer loop dependences (with distances smaller than the unroll factor), and the unrolling does not cause inner loop references to become misses (Figure 5.17).

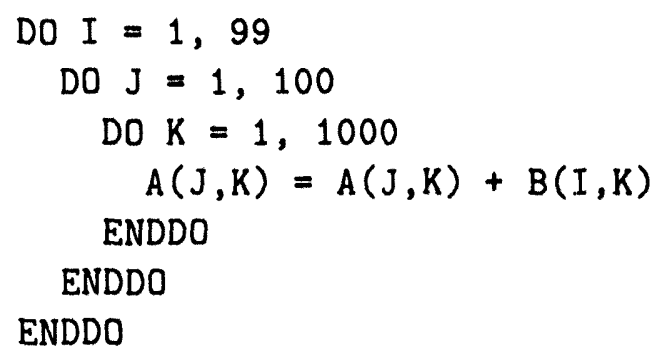

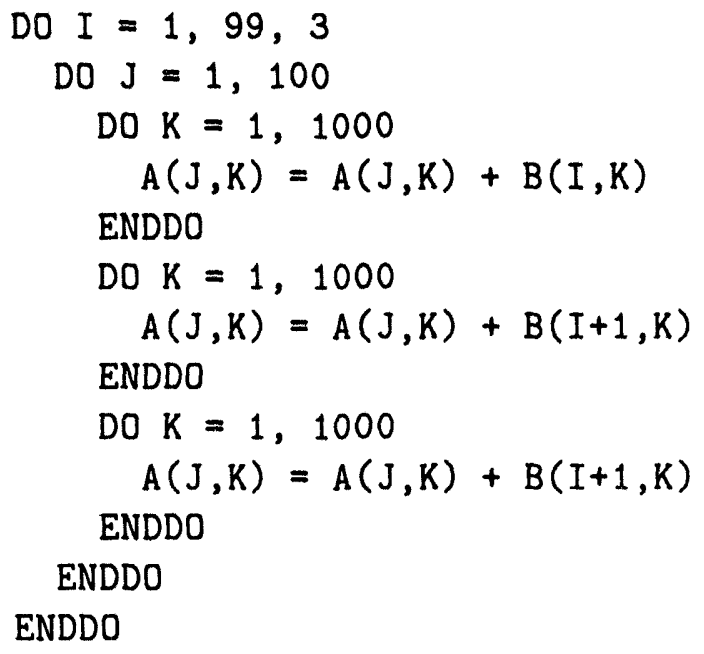

Figure 5.17: Example of Unroll and Jam

For a $32 \mathrm{~K}$ cache, the use of the $\mathrm{A}$ array will be a miss $9,900,000$ times. 'The 13 array will be a hit except to load each element (99,000 misses). The original program has 9,999,000 misses. After unroll and jam, the J loop carried dependences have a distance of one and remain hits. 'The first use of $\Lambda$ remains a miss, but the second and third uses become hits because the dependence is now loop independent. Only $3,300,000$ of the misses on A remain. Combined with the 99,000 misses from loading the $\mathrm{B}$ array, the unrolled and jammed version has 3,399,000 misses, just slightly over one-third of the original total. 


\section{CONCLUSIONS}

In this thesis, we addressed the need for novel performance metrics based on data motion. Results from the NAS parallel benchmarks and many other application programs show the failure of MFLOPS in predicting performance. For a performance model to be effective, it has to account for the time spent in moving data between the various levels of the memory hierarchy. We presented a simple model which uses the memory reference count of a program to predict its performance. To validate the model, experiments were conducted on a group of application programs and on various architectures. On the $n C U B E$, Sun $4 / 370$ and $\mathrm{PPSC} / 860$, this model was able to predict performance with about $\pm 50 \%$ accuracy. The model fails to predict the performance of $s f f t$ because of its high cache hit ratio.

To characterize the performance of hierarchical memory systems, a model based on reuse ratio was presented. We showed how the reuse ratio can be helpful in quantifying the compute intensity of algorithms.

A survey of some powerful program transformations was presented in the last chapter. These transformations are exploited during compiler memory management. They include loop fusion, loop distribution, loop interchange, loop skew, strip minc, unroll, strip mine and interchange, and unroll and jam. These transformations allow otherwise untransformable loop nests to be transformed into loops that are more 
amenable to increased memory performance.

\section{Future Work}

The performance model presented in this thesis accounts for only the memory reference count and ignores many other factors. One area of future research would be to work towards a more reliable performance metric that better accounts for the data motion. Moreover, in this thesis we assumed that only one processor is working on a problem. An increasingly popular method of increasing processing power is to supply multiple processors to perform the work. Understanding the memory performance of multiprocessors introduces several problems not present for uniprocessors. Methods to handle coherency issues and minimizing the memory invalidation traffic in the detection of memory references will be important. Projects like CEDAR are using multiple levels of cache to improve average memory latency. We need methods to use the compiler to tune the program for each level of the memory. 


\section{BIBLIOGRAPHY}

[1] R. Allen, Dependence Analysis for Subscripted Variables and its Applications to Program Transformation, Ph.D. Thesis, Rice University, Houston, 1983.

[2] J.R. Allen and K. Kennedy, Automatic loop interchange, Proceedings of the ACM SIGPLAN '84 Symposium on Compiler Construction, Montreal, Canada, June, 1984; SIGPLAN Notices, Vol. 19, No. 6, June, 1984, pp. 233-246.

[3] J.R. Allen and K. Kennedy, Automatic translation of Fortran programs to vector form, ACM transactions on programming languages and systems, Vol. 9, No. 4 , Oct. 1987, pp. 491-542.

[4] D. Bailey, J. Barton, T. Lasinski and H. Simon, The "NAS" Parallel Benchmarks, Report RNR-91-002, NASA/AMES Research Center, Jan. 1991.

[5] D. Callahan, A Global Approach to Detection of Parallelism, Ph.D. Thesis, Rice University, Houston, 1987.

[6] D. Callahan, J. Cocke, and K. Kennedy, Estimating interlock and improving balance for pipelined architectures, In 1987 International Conference on Parallel Processing, 1987.

[7] A. Charlesworth, High-locality parallel supercomputing, Document, FPS computing (now CRAY Research), Beaverton, Oregon, 1991.

[8] A. Charlesworth and J. Gustafson, Introducing replicated VISI to supercomputing: the FPS-164/MAX scientific computer, IEEE computer, Mar. 1986, pp. 10-23.

[9] J. Dongarra, J. Du Croz, S. Hammarling, and I. Duff, A set of level 3 basic linear algebra subprograms. ACM Trans. Math. Soft., Mar. 1990, pp. 1-17

[10] K. Gallivan, W. Jalby, U. Meier and $\Lambda$. Sameh, The impact of hierarchical memory systems on linear algebra algorithm design, Supercomputer Applications, Vol 2. No. 1, Spring 1988, pp. 12-48. 
[11] D. Gannon and W. Jalby, The influence of memory hierarchies on algorithm organization: Programming FFTs on a vector multiprocessor, In The (haracteristics of Parallel Algorithms, MIT Press, Cambridge, Mass., 1987.

[12] D. Gamnon. W. Jalby and K. Gallivan, Strategies for cache and local memory management by global program transformation, J. Parallel and Distributed Comput.. Vol. 5, 1988, pp. 587-616.

[13] ( .M. Goral, K.E. Torrance, D.P. Greenberg and B. Battaile, Modeling the interaction of light between diffuse surfaces, Comput. Craphics, Vol. 18, No. 3, July 1984.

[14] J.L. Gustafson Compute-intensive applications on advanced computer architectures, Procedings of the International Conference on Parallel Computing $9 /$. London. C.K., Sept. 1991 , pp. 75-89.

[15] J. Gustafson, D. Rover, S. Elbert and M. ('arter, The design of a scalable. fixedtime computer benchmark. J. of Parallel and Distributed Computing. Vol. 12. Aıg. 1991. pp. 388-401.

[16] J.L. Hennessy and D.A. Patterson. Computer Architecture: A Quan!itative Approach. Morgan Kaufmann, San Mateo. ('A, 1990

[17] L.B. Hostetler and B. Mirtich, DLXsim - A Simulator for DLX, , user mannal. via anonymous ftp from leland.stanford.edu.

[18] S.N. Kamin. Programing Languages: An Interpreter-Based Approach. ArddisonWesley, Reading. MA 1990.

[19] M.S. Lam. E.E. Rothberg and M.E. Wolf, The cache performance and optimizations of blocked algorithms, In Procedings of the Fourth International Conforence on Architectural Support for Programming Languages and Operating SiysIf m.s, Apr. 1991, pp. 6.3-7.4.

[20] L. Lamport, The parallel execution of DO loops, Comm. of the ACM, Vol. IT. No. 2. Feb. 1974.

[21] D.B. Loveman, Program improvement by source-to-source transformation. Journal of the ACM, Vol. 20, No. 1, Jan. 1977. pp. 121-145.

[22] A. Porterfield. Softurare Mrhods for Improvement of Cache Performaner on Supercomputer Applications. Ph.D. thesis, Rice Eniversity, Houston. May 1989. 
[23] W.J. Schmidt, Issues in the Design and Implementation of a Real-Time Ciarbage Collection Architecture, Ph.D. Thesis, lowa State University, Ames, 1992.

[24] J. Warren, A hierarchical basis for reordering transformations, In Procededings of the Elcuenth ACM Symposium on Principles of Programming Languages, 1984.

[25] M.E. Wolf and M.S. Lam, A data locality optimizing algorithm, In Proc. A( $\mathrm{Ml}$ SICIPLAN 91 Conf. Programming Language Design and Implementalion, Junc 1991 , pp. $30-44$.

[26] M.E. Wolf and M.S. Lam. A loop transformation theory and an algorithm to maximize parallelism, IEEE Trans. Parallel and Distributed Sys., Oct. 1991. pp. $452-471$.

[27] M. Wolfe, Optimizing Supercompilers for Supcrcomputers, Ph.1). Thesis, Iniversity of Illinois at Urbana-Champaign, Urbana, 1982

[28] M. Wolfe, Loop Skewing: the wavefront method revisited, Technical Report. Kunck and Associates, Inc. 1987.

[29] M.J. Wolfe, More iteration space tiling. Proc. Supercomputing 89, Nov. 1989. 


\section{APPENDIX A. DLX STANDARD INSTRUCTION SET}

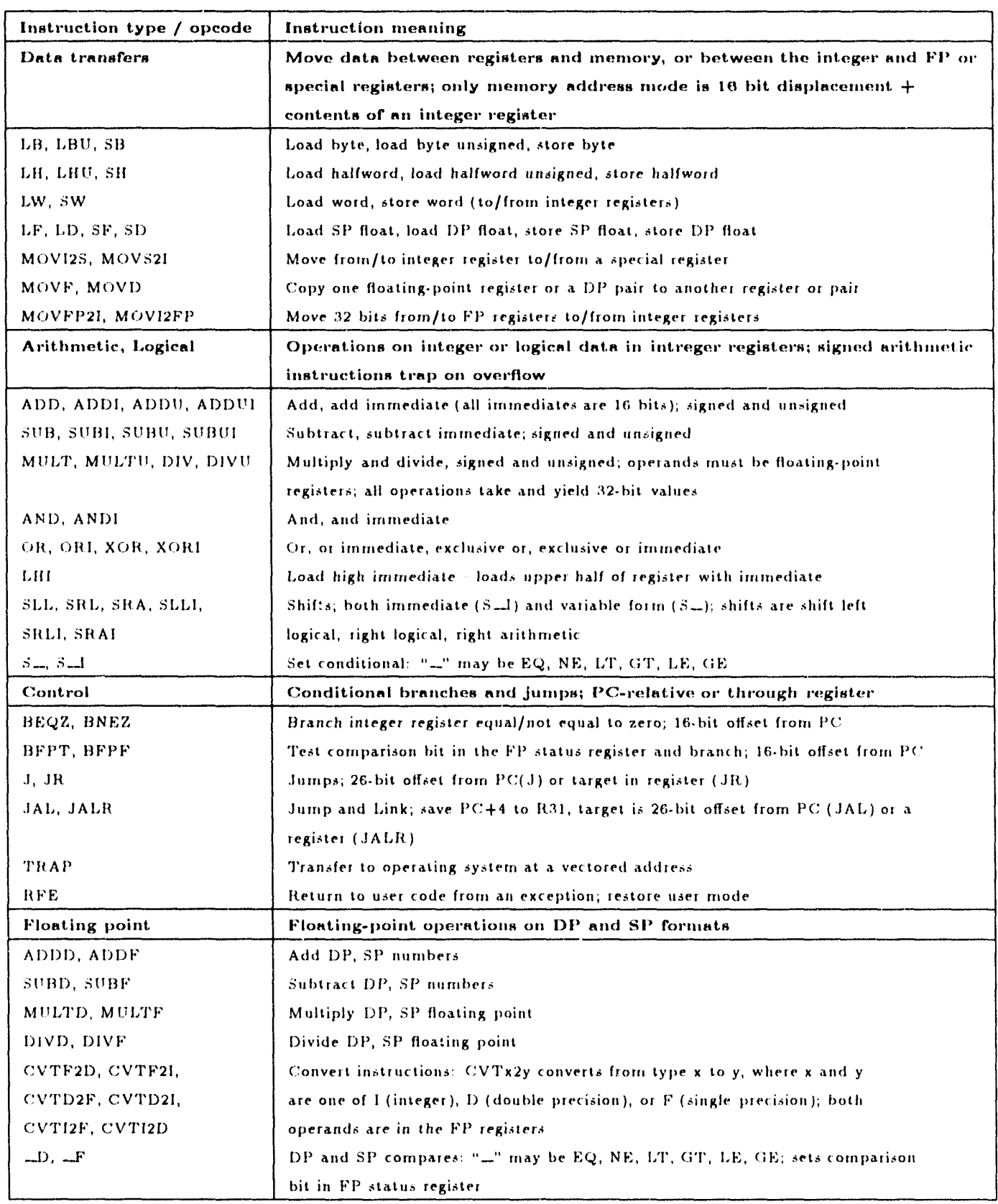




\section{APPENDIX B. DEPENDENCE THEORY}

Dependence theory is a method to describe the constraints on execution order of statements in a program. There are three kinds of data dependence between statements. The first kind occurs when a value computed (stored) in statement $S_{4}$ is used (fetched) in some statement $S_{w} . S_{w}$ is said to be data flow-dependent on $S_{1}^{\prime}$ and is written as $S_{v} \delta S_{u}$. This type of dependence relation shows how the data flows between the statements of the program. The second kind of data dependence occurs when an item is used in statement $S_{v}$ before that item is reassigned in some statement $S_{u} . S_{w}$ is said to be data anti-dependent on $S_{v}$, and is written as $S_{v} \delta S_{w}$. The last kind of data dependence occurs when an item is assigned in statement $S_{v}$ before that item is reassigned in some statement $S_{w} . S_{u}$ is said to be data output-dependent on $S_{v}$, and is written as $S_{v} \delta^{0} S_{w}$. Finally, $S_{w}$ is said to be control dependent on $S_{v}$, if execution of $S_{w}$ depends on the result of some conditional test in $S_{v}$, and is written as $S_{v} \delta^{c} S_{w}$.

Data dependences in the presence of DO loops can be characterized as either loop carried or looop independent [1]. A loop carried dependence is simply a dependence that exists between separate iterations of a loop. Each loop carried dependence is associated with a particular loop in the program. $A$ loop independent dependence is a dependence within a single iteration of the loop. Any dependence that is not. 
surrounded by a loop (i.e., exists if all loops are removed) is also loop independent.

We can also compute the dependence distances, and use them to describe the dependence arrows. For instance, in Figure B.1, the distances are 0 in the $I_{1}$ loop dimension, and +1 in the $I_{2}$ loop dimension. In this loop (as in most cases) the distances are small constants. Sometimes the distances are functions of one of the loop indices; other times the distances are unknown values, computed in the program. Thus, while the distance of the dependence is an exact way to describe the data dependence direction, it can be clumsy to find and represent in the general case.

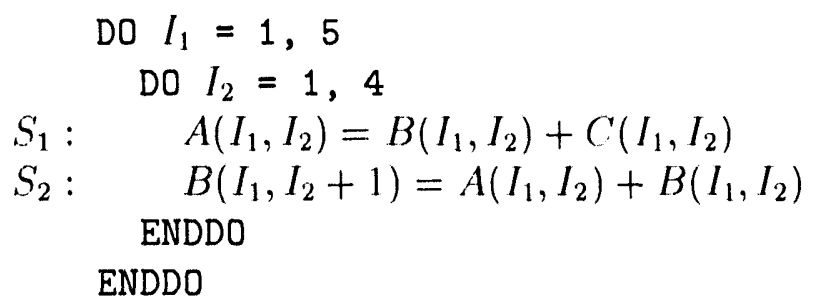

Figure B.1: Doubly-nested serial loop

A simpler but less exact description of the data dependence direction is the sign of the data dependence distance. The sign of the distance is positive if the dependence goes forward in that loop, negative if the dependence goes backward in that loop. and zero otherwise. The loop in Figure B.l has a data flow-dependence that goes forward in the $I_{2}$ loop.

In what follows, we assume loops that run forward, i.e., loops with positive increments. A data dependence direction vector is defined to be $\Psi=\left(\Psi_{1}, \Psi_{2}, \ldots, \Psi_{l}\right)$, a d-tuple of $d$ elements where $\Psi_{k} \in\{<,=,>, \leq, \geq, \neq, *\}$ and we say $S_{v} \delta_{\left(\Psi_{1}, \ldots, \Psi_{d}\right)}, S_{u}$ when 
1. there exist particular instances of $S_{v}$ and $S_{w}$, say $S_{v}\left[i_{1}, \ldots, i_{d}\right]$ and $S_{w}\left[j_{1}, \ldots, j_{d}\right]$, such that $S_{v}\left[i_{1}, \ldots, i_{d}\right] \delta S_{w}\left[j_{1}, \ldots, j_{d}\right]$, and

2. $i_{k} \Psi_{k} j_{k}$ for $1 \leq k \leq d$.

Each element of the data dependence direction vector corresponds to one of the loops that enclose the two statements in question. The direction in one dimension is independent of the directions in other dimensions. A forward direction ("<") means that the dependence crosses an iteration boundary forward (from iteration $i$ to iteration $i+1$ for example). A backward direction (">") means that the dependence crosses an iteration boundary backward (from iteration $i$ to $i-1$ ). An equal direction means that the dependence does not cross an iteration boundary. An asterisk ("**) is used when the direction is unknown, or when all three of $<,=,>$ apply. Data dependence direction vectors are a compact and convenient representation of the direction of the data dependence in the iteration space. 


\section{APPENDIX C. PROGRAM TO DETERMINE MEMORY \\ BANDWIDTH}

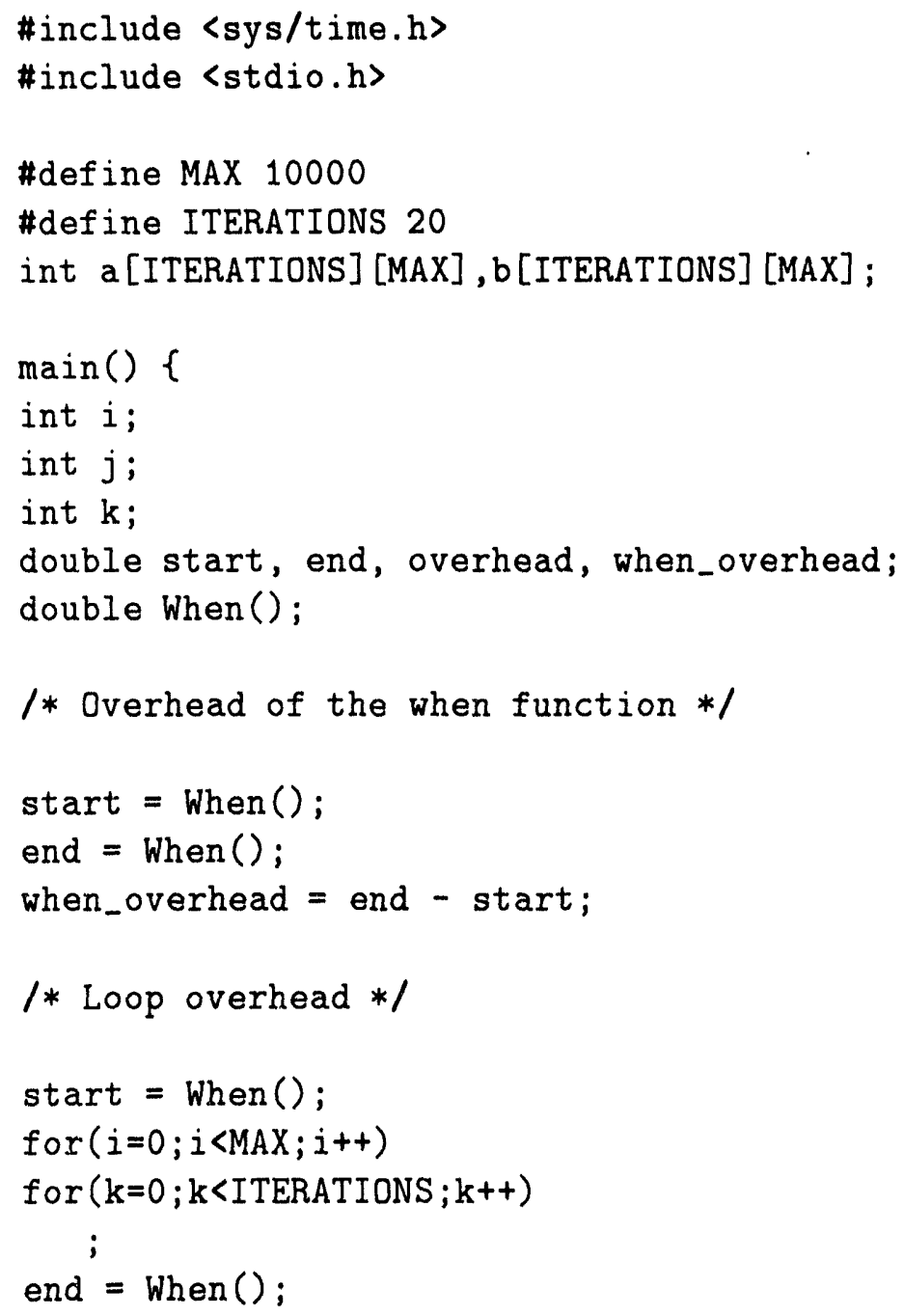




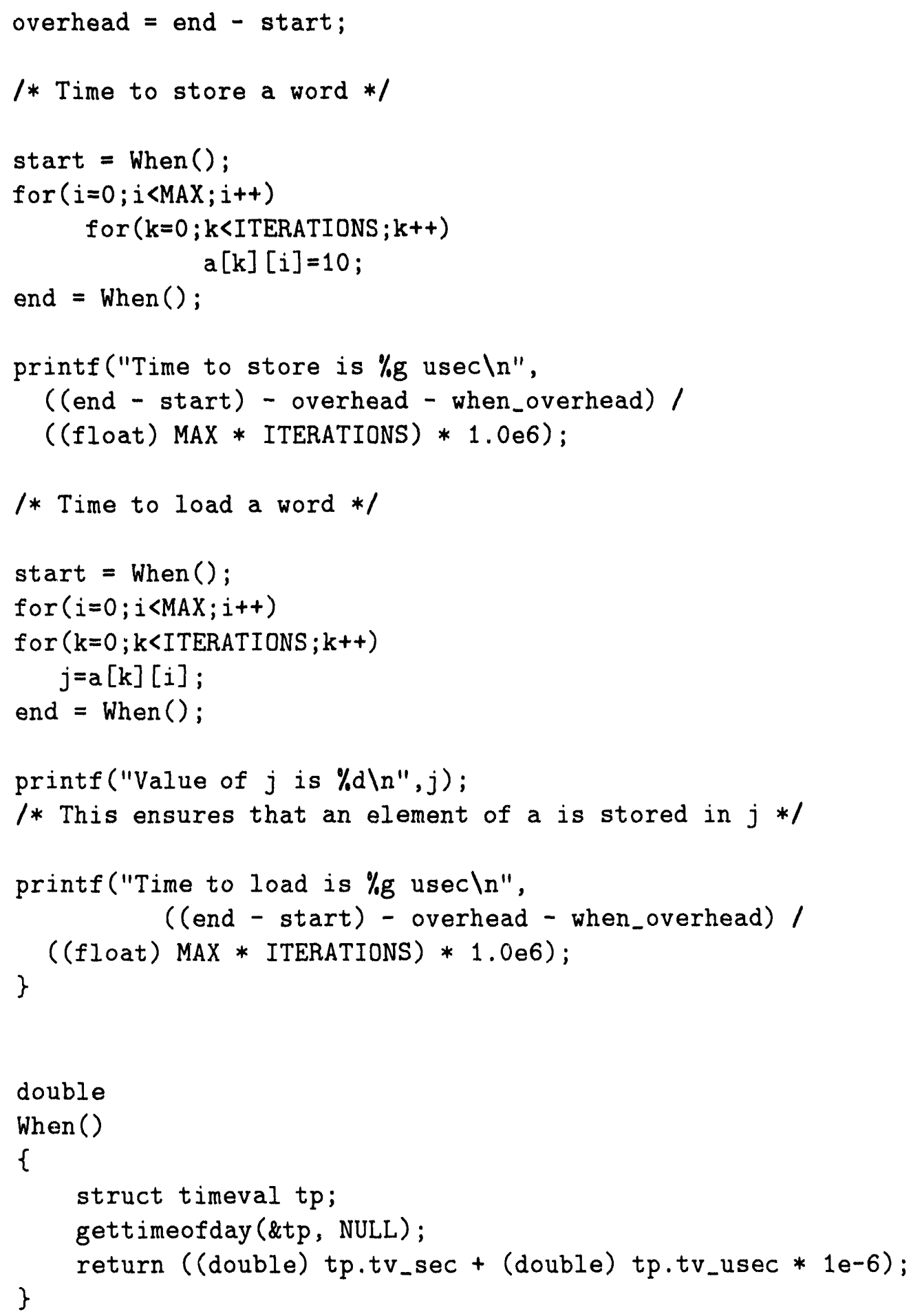



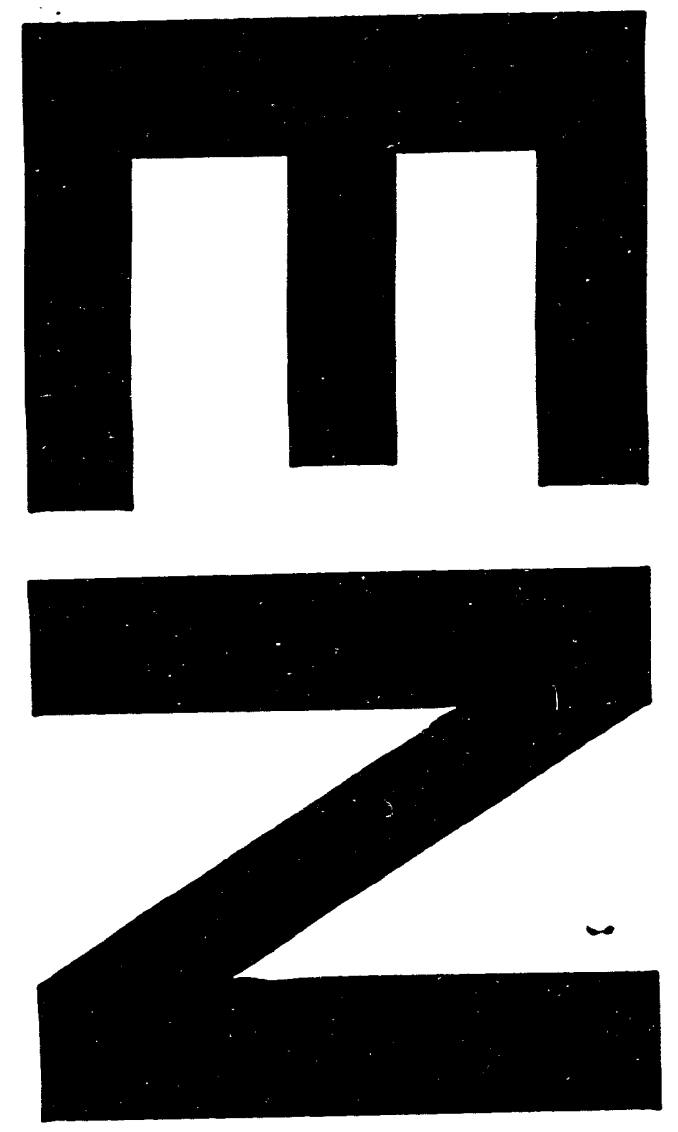

l
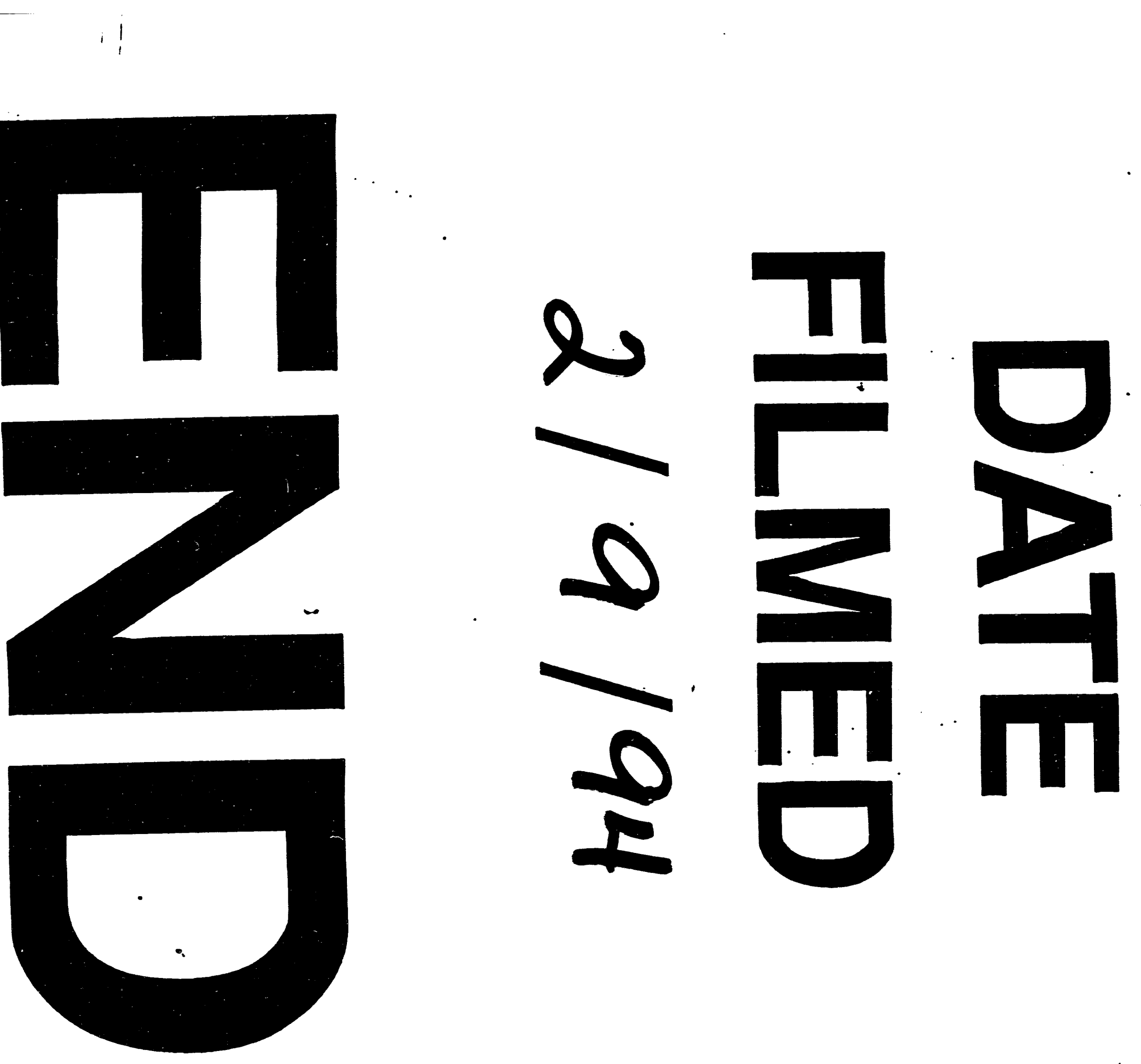
ב
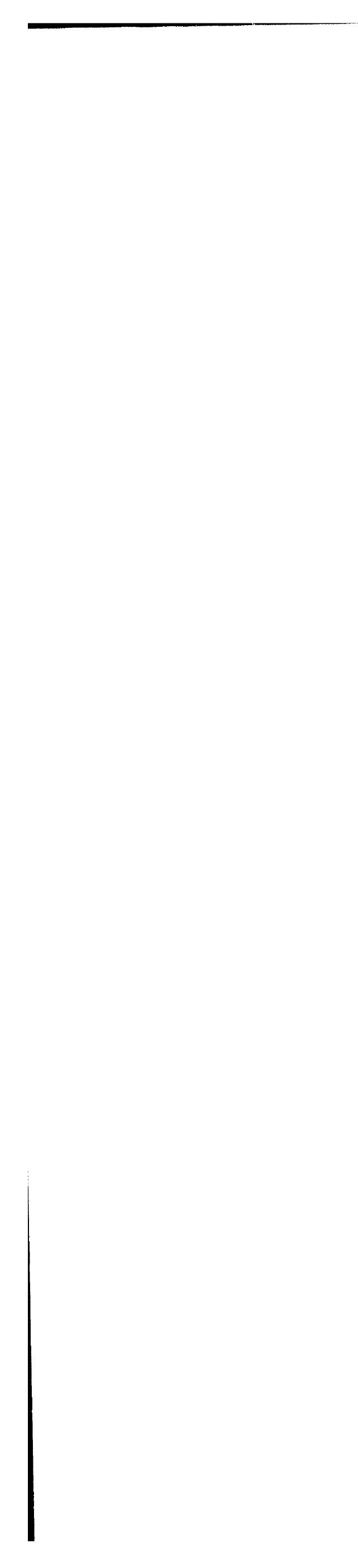